\title{
REVEALING SUBSTRUCTURE IN THE GALACTIC HALO: THE SEKBO RR LYRAE SURVEY
}

\author{
Stefan C. Keller, Simon Murphy, Sayuri Prior, Gary DaCosta, and Brian Schmidt \\ Research School of Astronomy and Astrophysics, Australian National University, Canberra, Australia \\ Received 2007 October 1; accepted 2007 November 14
}

\begin{abstract}
We present a search for RR Lyrae variable stars from archival observations of the Southern Edgeworth-Kuiper Belt Object survey. The survey covers $1675 \mathrm{deg}^{2}$ along the ecliptic to a mean depth of $V=19.5$, i.e., a heliocentric distance of $\sim 50 \mathrm{kpc}$ for RR Lyrae stars. The survey reveals 2016 RR Lyrae candidates. Follow-up photometric monitoring of a subset of these candidates shows $\sim 24 \%$ contamination by non-RR Lyrae variables. We derive a map of overdensity of RR Lyrae stars in the halo that reveals a series of structures coincident with the leading and trailing arms of debris from the Sagittarius dwarf galaxy. One of the regions of overdensity is found on the trailing arm, $200^{\circ}$ from the main body of the Sagittarius dwarf at a distance of $\sim 45 \mathrm{kpc}$. This distant detection of the stellar population of the outer trailing arm of Sagittarius offers a tight constraint on the motion of the dwarf galaxy. A distinctly separate region of overdensity is seen toward the Virgo overdensity.
\end{abstract}

Subject headings: Galaxy: halo - Galaxy: structure — stars: variables: other

Online material: color figures, machine-readable table

\section{INTRODUCTION}

The stellar halo of our galaxy offers a window to the past. Stars in the halo have long orbital periods and inhabit regions of space where the galactic potential is relatively smooth and slowly evolving. Consequently, structures in the halo suffer reduced spatial and kinematic dissipation and may persist for several gigayears (Bullock \& Johnson 2005). Such halo structures offer an insight into the process of galaxy formation, arguably one of the most important processes yet to be understood by modern astrophysics.

One schematic for the process of halo formation is that due to Eggen et al. (1962), who found that the stars on the most eccentric orbits were also the most metal-poor. This was interpreted as the signature of monolithic collapse of the protogalactic cloud, the most metal-poor, and hence oldest, stars forming during the collapse. This solitary process of galaxy formation was challenged by Searle \& Zinn (1978), who proposed the large dispersion seen in the metallicity of globular clusters and their lack of radial abundance gradient indicated that the Galaxy was the composite of a large number of subgalaxy-sized pieces.

Recent observational studies have converged on a combination of scenarios (Freeman \& Bland-Hawthorn 2002 for a review). This is perhaps most clearly demonstrated by the works of Kinman et al. (2007), Carollo et al. (2007), and Miceli et al. (2008), who find a distinct outer halo population. Carollo et al. (2007) shows that whereas the inner halo (galactocentric radius less than $15 \mathrm{kpc}$ ) is dominated by highly eccentric, prograde orbits and a metallicity of $[\mathrm{Fe} / \mathrm{H}] \sim-1.6$, the outer halo exterior to this possesses a more uniform distribution of eccentricities, includes highly retrograde orbits and $[\mathrm{Fe} / \mathrm{H}] \sim-2.2$. Kinman et al. (2007) study of the local halo similarly identifies a component that possesses retrograde rotation and streaming motions and another that exhibits negligible rotation and less signs of streaming. Kinman et al. furthermore show that the horizontal branch morphologies of the two components of the halo are consistent with those seen in the young and old globular clusters, reinforcing the conclusions of Lee \& Carney (1999) that the inner halo is consistent with rapid collapse and the outer halo with the ongoing accretion of dwarf galaxies.

A halo that formed from in situ star formation would possess, at the current epoch many dynamical times later, very little sub- structure. Simulations of galaxy formation in the context of $\Lambda \mathrm{CDM}$ predict that the majority of the stars in the outer halo formed in progenitor dwarf galaxies and were subsequently accreted (Abadi et al. 2006; Bullock \& Johnson 2005), with the general conclusion that significant amounts of substructure should survive to this time. However, we must point out that $\Lambda \mathrm{CDM}$ predictions on galaxy scales are contentious at this stage. Bell et al. (2007) compare the level of structure evident in the SDSS data with simulations and finds that the Milky Way's halo is well matched to a halo built exclusively from disrupted satellite galaxies.

Numerous studies of the spatial distribution of stars in the halo have shown the importance of ongoing accretion of satellite galaxies on to the Milky Way. The most striking example of ongoing accretion is the Sagittarius dwarf galaxy (Ibata et al. $1994 \mathrm{Sgr}$ ). The ensuing trail of debris has been found to wrap around the sky (Belokurov et al. 2006; Majewski et al. 2003; Ibata et al. 2003; Yanny et al. 2003; Newberg et al. 2002). The debris of Sgr is arguably the largest contributor yet found to the halo substructure at radii of over $50 \mathrm{kpc}$. The orbit of $\mathrm{Sgr}$, as traced by its extensive debris tails, has been used as a probe of the mass and shape of the Galaxy (Ibata et al. 2001; Helmi \& White 2001; Helmi 2004; Johnston et al. 2005; Law et al. 2005; Fellhauer et al. 2006).

Another halo structure, the Virgo stellar stream (VSS) was found as an overdensity of RR Lyrae stars (RRLs) (Vivas \& Zinn 2006; Zinn et al. 2004) at galactocentric radius $\sim 20 \mathrm{kpc}$. Later radial velocity measurements (Duffau et al. 2006) revealed the grouping shared a common space velocity. Newberg et al. (2002), Jurić et al. (2005), and more recently Newberg et al. (2007) find a coincident diffuse overdensity of F-type main-sequence stars spanning some $1000 \mathrm{deg}^{2}$ of sky called the Virgo overdensity (VOD). Newberg et al. (2007) proposes that the VSS and VOD are the same structure. On the basis of radial velocity measurements Newberg et al. concludes it is not associated with a confluence of the leading and trailing arms of Sgr, as proposed by MartínezDelgado et al. (2007), but is possibly remnant material from another merger event.

The Monoceros stream (Newberg et al. 2002) is believed to be a low surface brightness ring encircling the Galaxy at 15-20 kpc (Ibata et al. 2003). The structure was first traced by Newberg et al. (2002) using halo turnoff stars and subsequently by Majewski 
TABLE 1

Wide-Field SuRVEYS FOR RRLs

\begin{tabular}{|c|c|c|c|}
\hline Survey Name & $\begin{array}{l}\text { Sky Coverage } \\
\left(\mathrm{deg}^{2}\right)\end{array}$ & Number of Candidates & $\begin{array}{c}\text { Maximum Distance } \\
(\mathrm{kpc})\end{array}$ \\
\hline 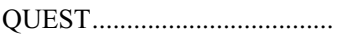 & 380 & 498 & 55 \\
\hline 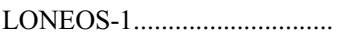 & 1430 & 838 & 30 \\
\hline Ivezić et al. (2000).................... & 100 & 296 & 65 \\
\hline 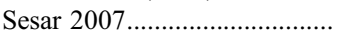 & 290 & 634 & 100 \\
\hline Current survey ............................ & 1675 & 2016 & 50 \\
\hline
\end{tabular}

et al. (2003) using M giants. The stream is not thought to be related to Sgr tidal debris or the VSS/VOD, but rather may result from the disruption of the Canis Major dwarf (Martínez-Delgado et al. 2007; Casetti-Dinescu et al. 2006). Conn et al. (2007) reports that interpretation of the Monoceros stream as a warp or flare in the galactic disk does not account for the observed stellar densities. Possibly related to the Monoceros Stream are the Tri-And overdensity (Rocha-Pinto et al. 2004) at $20 \mathrm{kpc}$ and a yet more distant stellar structure at $\sim 28 \mathrm{kpc}$ (Martin et al. 2007).

Most recently, Belokurov et al. (2007) reports the detection of a significant stellar overdensity that covers some $80^{\circ}$ of galactic latitude, dubbed the Hercules-Aquila Cloud. This overdensity lies at a distance of between 10-20 kpc and straddles the Galactic disk offset by $6-15 \mathrm{kpc}$ from the Galactic center. Spectroscopy of probable cloud members shows that the structure possesses a radial velocity offset of $\sim 180 \mathrm{~km} \mathrm{~s}^{-1}$ from the halo and thick disk.

The features discussed above are a small fraction of the number that could be expected from simulations of $\Lambda \mathrm{CDM}$ hierarchical structure formation (Bullock et al. 2001; Bullock \& Johnson 2005). The search for relic substructure could help illuminate the outstanding "missing satellite problem" in galaxy formation in $\Lambda \mathrm{CMD}$ cosmology (Freeman \& Bland-Hawthorn 2002). Here, models predict of order 500 small dark matter halos should surround the Milky Way today, in contrast to the $\sim 40$ known dwarf galaxies in the Local Group (Wilkinson 2007). If the level of substructure does not match that predicted from a large population of dwarf galaxies this may prompt revisions to model assumptions.

\section{RR LYRAE VARIABLES AS PROBES OF THE HALO}

RRL variables have a long history as probes of the Galactic halo (see Wetterer \& McGraw [1996] for a historical review). They are stars on the core He-burning horizontal branch that fall within the temperature range of the instability strip. They are easily identified on the basis of their color and variability. In addition, their absolute magnitudes are known to better than 0.1 mag, making them standard candles for the halo. They represent an old stellar population and so by virtue of their age can probe disruption events that occurred at early times. The abundance of RRLs is dependent on the morphology of the horizontal branch of the progenitor population. Local dwarf spheroidals exhibit abundant RRL populations, hence these variable stars should be good tracers of accretion events.

In recent years several sky surveys have discovered large numbers of RR Lyrae variables. The survey area, limits and completeness of these surveys are summarized in Table 1. The QUEST RRL survey (Vivas \& Zinn 2006) proposes six significant substructures; three associated with the Monoceros stream, and others associated with Pal 5, VSS, and the Sagittarius stream. Searches for RRLs in the SDSS are presented by Sesar (2007) and Ivezic et al. $(2000,2004)$ from multiple imaging and by Ivezić et al.
(2005) from colors alone. Sesar (2007) reviews the SDSS work to date, proposing 13 new overdensities.

The QUEST and SDSS RRL surveys cover equatorial regions. The LONEOS-1 Survey (Miceli et al. 2008) traces the ecliptic but to a moderate depth (reaching distances of up to $30 \mathrm{kpc}$ ). With between 28-50 epochs per star Miceli et al. (2008) are able to identify the Oosterhoff type of each RRL. They conclude that the radial distribution of Oosterhoff type I and II (OoI and OoII) RRLs are systematically different. Lee \& Carney (1999) found globular clusters that are dominated by OoI RRLs show little net angular momentum as expected from a formation scenario such as Searle \& Zinn (1978), whereas OoII globular clusters show net prograde rotation as would result from the Eggen et al. (1962) scenario. Miceli et al. (2008) propose that the presence of distinct OoI and OoII populations reveals that both the monolithic collapse and accretion scenarios are important for constructing the halo. This conclusion parallels the two kinematic components found in the halo by Carollo et al. (2007). The presence of substructure in their sample of RRLs is not addressed in Miceli et al. (2008).

\section{THE SOUTHERN EDGEWORTH-KUIPER BELT OBJECT SURVEY}

The present study is based on the archived observations of the Southern Edgeworth-Kuiper Belt Object (SEKBO) survey (Moody et al. 2003). SEKBO was a multiepoch, two-color imaging survey of a $10^{\circ}$ band centered on the ecliptic. Observations were obtained over the 3 years from December 1999 on the Mount Stromlo Observatory 50 inch (1.27) Great Melbourne Telescope that was previously used for the MACHO microlensing survey. The telescope featured a dual-band imaging system in which a dichroic allowed for simultaneous exposures in two broad bands on separate $4 k \times 4 k$ pixel CCD mosaics. The MACHO blue filter, hereafter $B_{\mathrm{M}}$, spans $455-590 \mathrm{~nm}$ (wavelengths at 50\% normalized response) and the red, $R_{\mathrm{M}}$, from 615-775 $\mathrm{nm}$ (Bessell \& Germany 1999). These wide, nonstandard filters most closely match Johnson $V$ and $R$.

SEKBO was designed to search for trans-Neptunian objects from a set of three exposures of a given field, the first and second epochs separated by $\sim 4 \mathrm{hr}$ and a subsequent third epoch 1-7 days later. As the telescope operated in an automated manner with limited environmental monitoring some observations were obtained under conditions of insufficient quality. These would break an observing sequence for a field and require reacquisition of the field at a later date. Figure 1 shows the distribution of time intervals between repeat observations of the fields in our sample. The exposure time was $300 \mathrm{~s}$. Under reasonable photometric conditions, this results in a useful magnitude range for photometry of $14<V<20$. Figure 2 shows the distribution of seeing derived from the SEKBO images. The seeing is typically $2^{\prime \prime}$ with a tail out to $>5^{\prime \prime}$. 


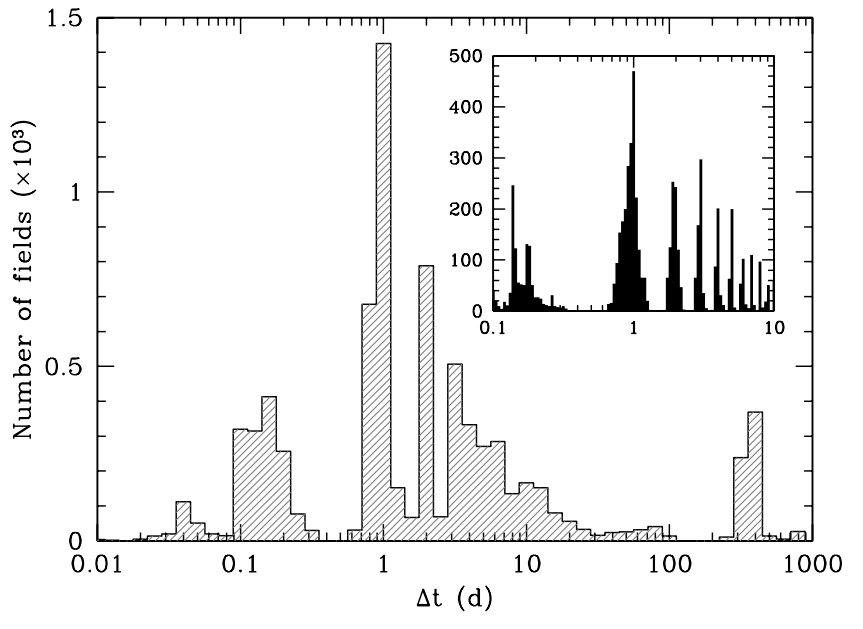

FIG. 1.-Time difference in days between epochs for the SEKBO fields. As expected from the survey design there are clusters of observations at $1-4 \mathrm{hr}$ and 1-7 days apart. The group at $1 \mathrm{yr}$ are repeat observations of fields that failed the previous year. The insert shows a the distribution between $0.1-10$ days.

There are 5212 field centers with two or more epochs that form the basis of our sample. The histogram of the number of epochs per field in the sample is shown in Figure 3. Most fields have 2 or 3 observations, but there is a tail out to 10 observations. Figure 4 shows the sky coverage of the survey. Note that we have excluded regions with $E(B-V)>0.10$ as well as galactic latitudes $b<$ $10^{\circ}$ to avoid fields with excessive crowding. The total effective area of the survey (having corrected for inter-CCD gaps and bad columns; see S6) is $1675 \mathrm{deg}^{2}$.

\section{PHOTOMETRIC REDUCTION}

In this section we outline the steps taken to reduce the raw frames to calibrated photometry. Each of the 17578 exposures

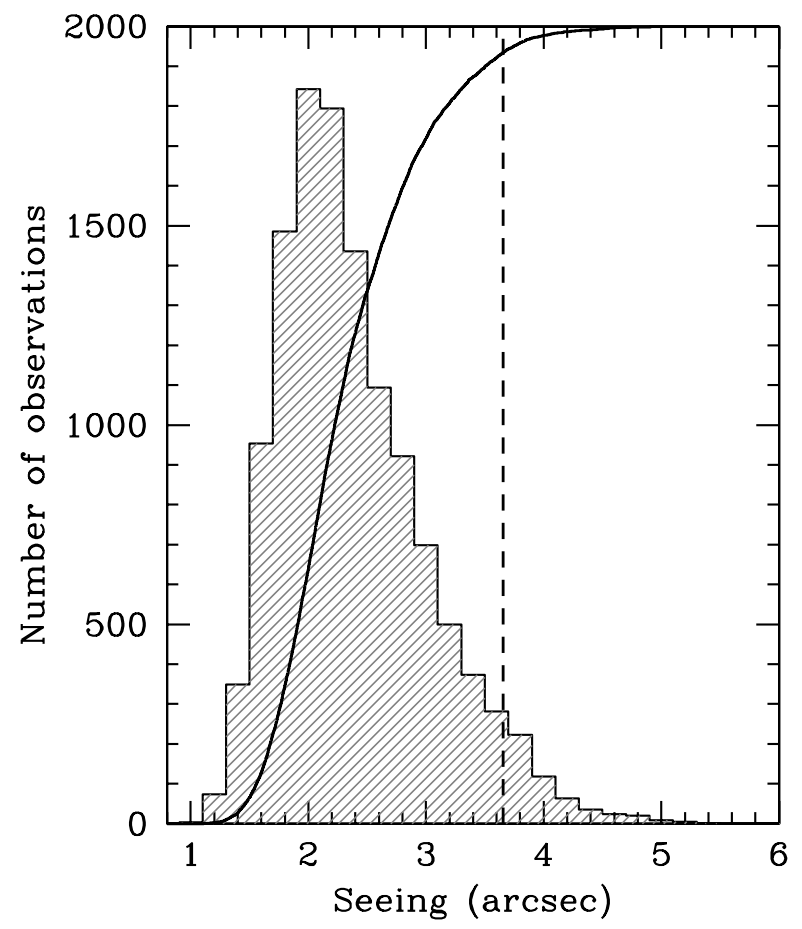

FIG. 2.- Seeing distribution of images in the present study. The solid line is the cumulative distribution, while the dashed line shows the 3.6" seeing cut imposed on the observations, as detailed in $\S 4.2$

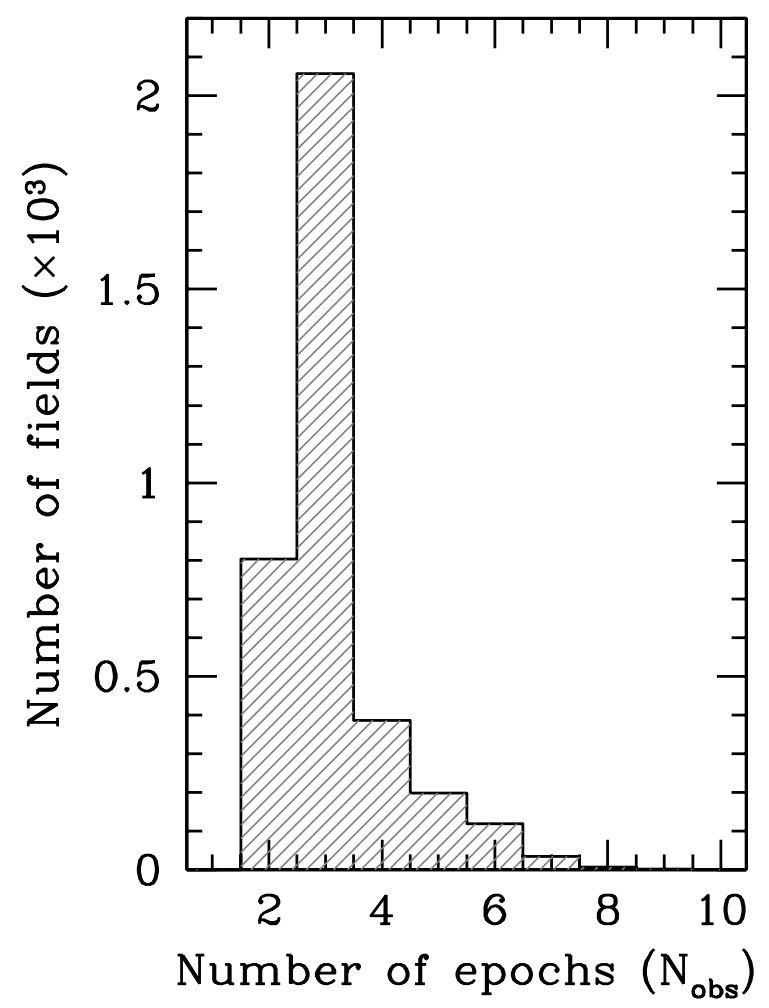

FIG. 3.-Histogram of the number of images per field for the sample of 5212 fields.

considered consists of 16 images (two colors $\times$ four CCDs $\times$ two amplifiers), which are stored on the Australian National University's Supercomputing facility mass data store. The data set is 2.3 TB in size.

The data in the archive has been processed to remove bias and flat-field structure using the data reduction pipeline of the MACHO system. This system maintained a moving average bias and flat field that was regularly checked for changes in the optical/detector system (Alcock et al. 1999).

We first determine a world coordinate system (WCS) for each image based on the nominal pointing of the telescope as recorded in the image FITS header. UCAC2 stars (Zacharias et al. 2004) in common to the image are used to derive the WCS. The UCAC2 provides between 40 and several hundred stars per CCD. Our derived positional uncertainties are better than 50 mas for bright stars. A ZPN ${ }^{1}$ coordinate representation is used. This representation utilizes a radial term to characterize distortions across the image and a simple linear transformation to remove the effects of rotation, scale, and any shear caused by refraction. Linearizing the telescope system in this way provides robust coordinate transformations across multiple images of a field with no difficulties in CCD corners or in images with few astrometric standards.

Differences in the fabrication and electronics associated with each CCD/amplifier pair result in a nonuniform gain across the red and blue mosaic. Gain matching between each CCDs two amplifiers was achieved by matching the median sky background level. The four CCDs were then mosaicked together using the SWarp package. ${ }^{2}$ The median sky brightness was then used to scale the four CCDs of each passband to the same gain level.

\footnotetext{
${ }^{1}$ Zenithal/azimuthal polynomial projection; see Calabretta et al. (2004).

2 See http://terapix.iap.fr, Bertin (2005).
} 


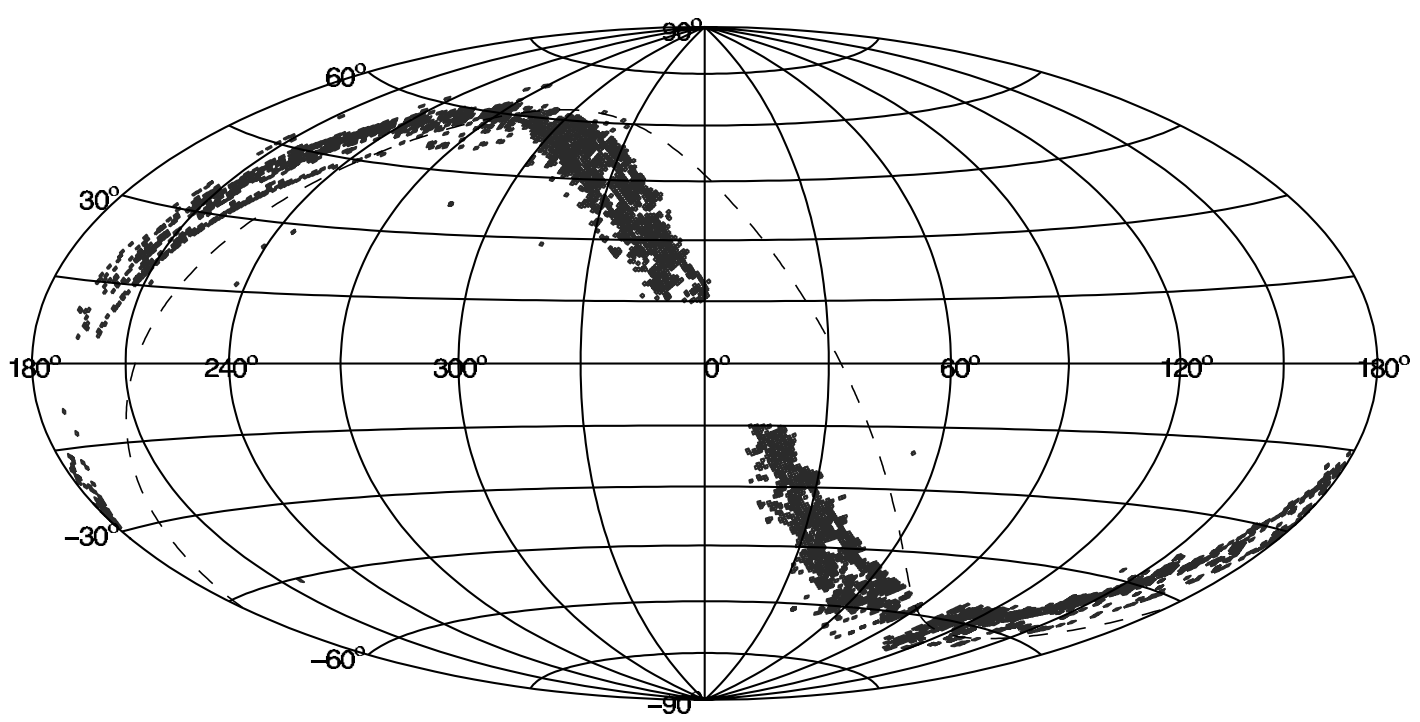

FIG. 4.-Distribution of fields in the present survey in galactic coordinates. The dashed line is the celestial equator.

\subsection{Photometry and Object Classification}

We base our object catalog on a template image that is created for each field by co-adding the individual $R_{\mathrm{M}}$ observations. In the case of three or more images we use SWarp to form a median image to robustly reject bad pixels and cosmic rays. In the case of two images for a field we apply an algorithm that examines corresponding pairs of pixels in the registered image stack and removes high values from pairs with large variance. The footprint of the template image is only that area on the sky that is represented in all images of the field.

The template image is used solely for object detection. The combination of images taken under different seeing would render the extraction of photometry from the template problematic due to the distorted and nonphotometric point-spread function (PSF) shape that results from combination. The template image does have several advantages for object detection, however, including greater depth and reduced numbers of false objects due to the exclusion of cosmic rays and bad pixels. Object detection within the template image was made using the cdoPhot ${ }^{3}$ package.

Photometry was then performed on the individual images that comprised the template. This photometry was performed in three modes: in fixed position aperture mode where the template coordinates are used for the center of the aperture; centroid aperture mode where the barycenter of the object is used for the center of the aperture; and PSF-fitting mode where a PSF is constructed for each object. Aperture corrections were applied to correct the magnitude defined through a 3 pixel radius aperture to the aperture used by Landolt (1992) for standard star photometry (discussed below).

The PSF-fitting mode is the basis of our object classification. The Gaussian widths in the $x$ - and $y$-directions give a clear discriminant as to the nature of the object. Stellar objects lie clustered around a particular $x$-width, $y$-width. Galaxies exhibit more extended dimensions and cosmic rays a smaller extent. To make a quantitative judgment as to the stellar nature of the objects in our data we construct a stellarity index that expresses the distance an object lies in Gaussian $x$-width, $y$-width space from the stellar locus.

\subsection{Standardized Instrumental Photometry}

Raw magnitudes from the gain matched frames were then placed on a standard instrumental system. Due to nonphotometric conditions and atmospheric extinction there were zero-point offsets between magnitudes in each observation and that obtained from the detector system at the zenith under photometric conditions (hereafter the standard instrumental system). The 2MASS (Skrutskie et al. 2006) $J$ and $K$ magnitudes allow this offset to be calculated. From images obtained in photometric conditions we first derived a linear fit:

$R_{\mathrm{M}, i}-J=-2.5 \log f_{R_{\mathrm{M}, i}}+2.5 \log t_{\mathrm{exp}}-J=\alpha(J-K)+\mathrm{ZP}_{R_{\mathrm{M}, i}}$

where $f_{R_{\mathrm{M}, i}}$ is the flux in MACHO $R$ for the $i$ th observation, $t_{\text {exp }}=300 \mathrm{~s}$ and $\mathrm{ZP}_{R_{\mathrm{M}, i}}$ is a zero point defined below. We determine $\alpha=1.100$ over a narrow color range $(0.3<J-K<0.6)$.

For each individual image in the data set, a fit was then performed on the order of 100 stars in each field with signal-to-noise ratio $>20(J<15$ and $K<14.5)$ within the color range above. We define our standard instrumental system such that a $R_{\mathrm{M}}=$ 22 star provides $1 \mathrm{ADU} \mathrm{s}^{-1}$. Hence:

$$
R_{\mathrm{M}, i}-\mathrm{ZP}_{R_{\mathrm{M}, i}}=22
$$

where $\mathrm{ZP}_{R_{\mathrm{M}, i}}$ is the zero point required to draw the observation onto the standard instrumental system $\left(\mathrm{ZP}_{R_{\mathrm{M}, i}}\right.$ is the extrapolation of the linear relation of equation (1) to $J-K=0$ ).

The effective wavelength of the $B_{\mathrm{M}}$ filter is sufficiently blue of the 2MASS bands that the above procedure cannot be used as it would introduce a significant interstellar extinction dependence. To determine the $B_{\mathrm{M}}$ zero point we used the $V-R$ color of sources from Stetson (2000) and their corresponding $B_{\mathrm{M}}-R_{\mathrm{M}}$ colors. Note that because the blue and red exposures are obtained simultaneously there is a single offset between $B_{\mathrm{M}}$ and $R_{\mathrm{M}}$ (with a minor air mass dependence, see below) and this is found by the requirement that

$$
V-R=0=\left(B_{\mathrm{M}}-\mathrm{ZP}_{B_{\mathrm{M}}}\right)-\left(R_{\mathrm{M}}-\mathrm{ZP}_{R_{\mathrm{M}}}\right) .
$$




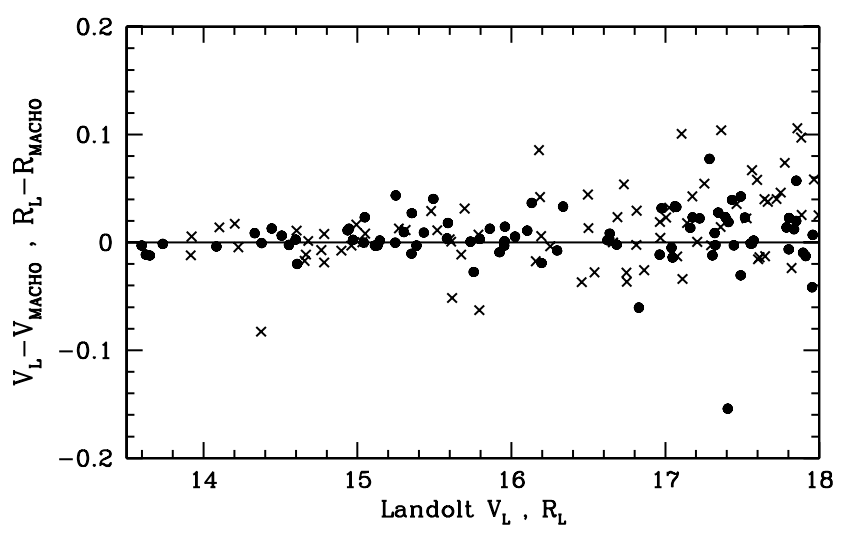

FIG. 5.-Difference between the Landolt $V$ (circles) and $R$ (crosses) standard magnitudes and those derived from our photometry for the Stetson (2000) stars in our sample.

Since the MACHO filters are well matched to the standard $V$ and $R$ only a linear regression is required. However, due to the chromatic effect of atmospheric extinction, the difference in zero points will be a function of air mass, $X$. From the observed Landolt fields we derive

$\mathrm{ZP}_{B_{\mathrm{M}}}-\mathrm{ZP}_{R_{\mathrm{M}}}=(0.316 \pm 0.06)-(0.08 \pm 0.02)(X-1)$

and scale the flux of the blue images to provide $1 \mathrm{ADU} \mathrm{s}^{-1}$ for a $B_{\mathrm{M}}=22$ star.

We reject those observations displaying zero-point magnitudes that show more than 0.75 mag of extinction or sky brightness that are more than 1 mag brighter than the median. We also remove those observations with seeing in excess of $3.6^{\prime \prime}$ to reduce the problems introduced into the RRL detection technique by objects that are blended in poorer seeing frames and resolved in nominal seeing frames. This leaves 3692 field centers with acceptable data.

\subsection{Transformation to $V$ and $R$}

A number of fields in the data set contain standard stars observed by Stetson (2000). This allows us to define the transformation equations to take our standard instrumental photometric system to that of Landolt $V$ and $R$ (Landolt 1992). Given the close matching of the MACHO bandpasses to the $V$ and $R$ filters only a linear transformation in color is required. The transformation is of the form

$$
V=B_{\mathrm{M}}+\alpha\left(B_{\mathrm{M}}-R_{\mathrm{M}}\right)+\beta
$$

for constants $\alpha$ and $\beta$, and where $B_{\mathrm{M}}$ and $R_{\mathrm{M}}$ have been both zero-point offset and aperture corrected. Observations of a large sample of standard stars allow us to define the following conversions to Landolt $V$ and $R$ magnitudes:

$$
V=B_{\mathrm{M}}-0.12\left(B_{\mathrm{M}}-R_{\mathrm{M}}\right)+0.19,
$$

and

$$
R=R_{\mathrm{M}}+0.15\left(B_{\mathrm{M}}-R_{\mathrm{M}}\right)+0.34 \text {. }
$$

Figure 5 shows the difference between the magnitudes in $V$ and $R$ derived from equation (6) and those of Stetson (2000) from the 160 stars in common. This figure shows an offset of $\Delta V=0.01 \pm 0.03$ and $\Delta R=0.01 \pm 0.03$. It should be noted

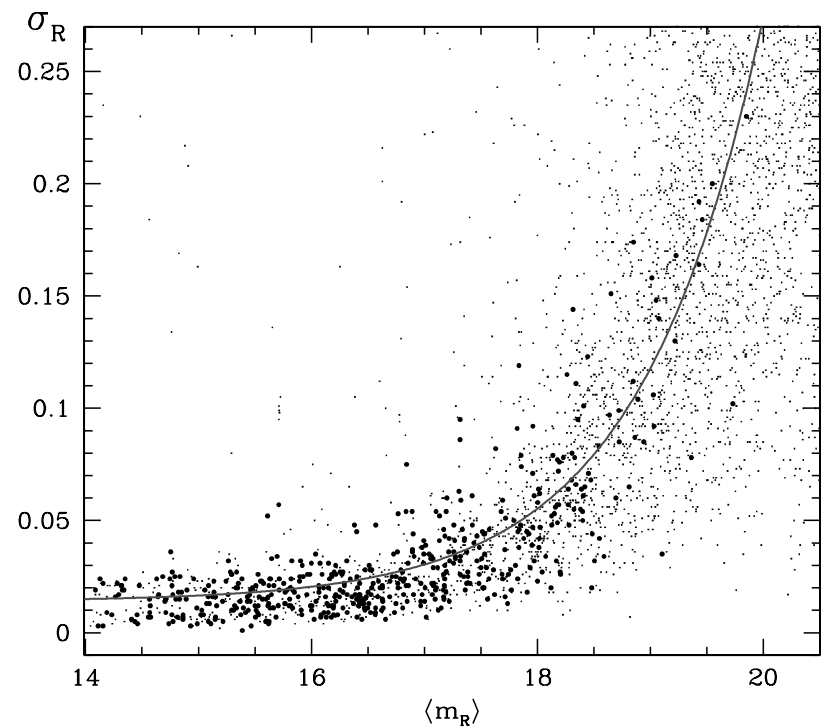

FIG. 6.-Standard deviation of the photometry in $R_{\mathrm{M}}$ as a function of magnitude for typical field in our survey. Overlaid is the sky-limited Poisson curve (line; see text) that is a fit to objects with unambiguously stellar shape parameters (bold points).

that the photometry that contributes to Figure 5 was obtained under heterogeneous seeing, atmospheric extinction and photometric conditions over the course of 3 years giving us confidence in our global zero points to the level of $\pm 0.03 \mathrm{mag}$.

\section{RR LYRAE CANDIDATE SELECTION}

We have implemented a probabilistic approach to the selection of RRL candidates. We first remove those objects with shape and color parameters grossly unlike the stellar population. Selection is then made based on color and variability. In order to quantify the significance of variation we need to first understand the photometric uncertainties.

Photometric uncertainties were characterized using the observed standard deviations of the stellar objects from the multiple epochs. Figure 6 shows the distribution of standard deviation versus magnitude for objects in a particular survey field. For most stars the standard deviations are indicative of the photometric uncertainties for the observation. However, variables stars or objects with bad photometry on some epochs will lie above the main locus.

To determine the mean photometric uncertainty as a function of magnitude we fit a sky-limited Poisson distribution:

$$
\sigma_{\text {phot }}=10^{+0.4\left(m+m_{\text {cut }}\right)}+m_{\text {floor }}
$$

for magnitude $m$, where $m_{\text {cut }}$ is iteratively determined so that the standard deviation of half the objects lie above the fitted line and half below. The constant $m_{\text {floor }}$ is the component of the photometric uncertainty that is not related to the photon statistics. This is derived from the brighter stars up to 2 mag fainter than the saturation limit. The photometric uncertainties vary from field to field. The typical uncertainty for the brighter stars is $0.02 \mathrm{mag}$ rising to 0.2 mag at $V \sim 19.5$.

With an understanding of our photometric uncertainties we then construct a RRL index, $\mathcal{L}$ :

$$
\mathcal{L}=\mathcal{L}_{\text {color }}+\mathcal{L}_{\text {RRL }}-\mathcal{L}_{\text {not var }}
$$

where $\mathcal{L}_{\text {color }}$ quantifies the probability that a star has colors commensurate with an RRL; $\mathcal{L}_{\mathrm{RRL}}$ is the log likelihood that the 


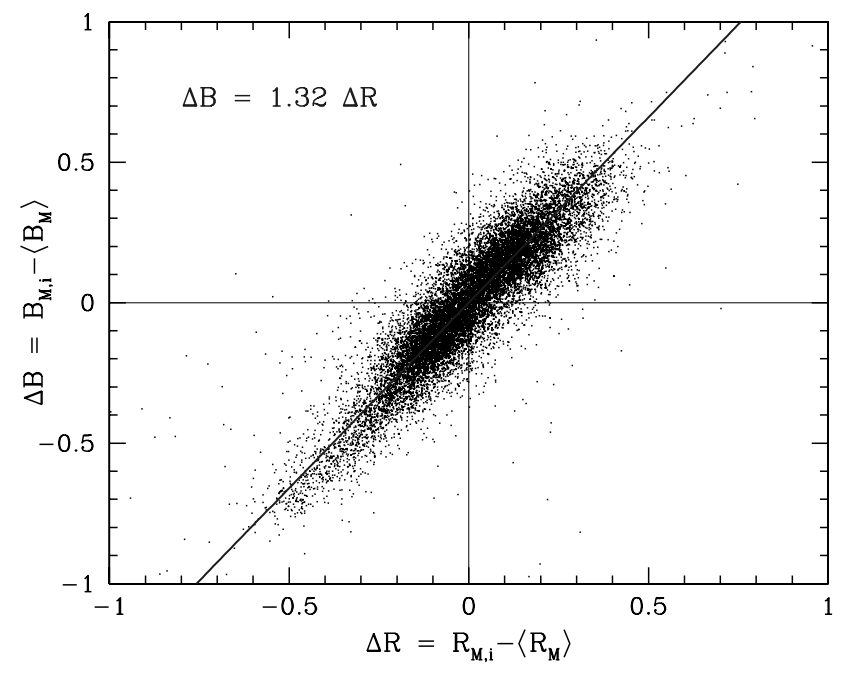

FIG. 7.- Observed color variation of a sample of 119 MACHO RR Lyrae from the LMC. The line shows the best-fit relation $\Delta B=1.32 \Delta R$.

variation in color exhibited by the object matches that expected from RRLs; and $\mathcal{L}_{\text {not variable }}$ is the log likelihood that the variation can be explained by photometric uncertainty alone. The RRL index, $\mathcal{L}$, is a figure of merit of how much like an RRL each object is. It is not based on a deep statistical foundation, but rather the pragmatic need to distinguish RRLs from other stars with a minimum of observations and with minimal manual intervention.

We now discuss the above three quantities in more detail. To construct $\mathcal{L}_{\text {color }}$ we first derived the de-reddened color distribution from $\sim 19000$ LMC RRLs from the MACHO project [using a mean reddening of $E(V-R)=0.08$ toward the LMC; Alcock et al. 2004]. This distribution can be approximated by a Gaussian with $\langle V-R\rangle_{0}=0.137$ and $\sigma_{V-R}=0.08 \mathrm{mag}$. Given this distribution the probability density of a star having an RRL-like color is

$\mathcal{L}_{\text {color }}(V-R)_{0, i}=\frac{1}{\sigma_{V-R} \sqrt{2 \pi}} e^{-1 / 2\left[(V-R)_{0 i}-\langle V-R\rangle_{0 / \sigma_{V-R}}\right]^{2}}$,

where the dereddened $V-R$ color of the $i$ th observation, $(V-R)_{0 i}$, is derived from equation (6) and dereddened according to the dust maps of Schlegel et al. (1998) [we adopt $E(V-R)=0.608 E(B-V)$ and $\left.A_{V}=3.315 E(B-V)\right]$.

$\mathcal{L}_{\mathrm{RRL}}$ compares the observed variation for an object to the variations seen from a sample of RRLs. Densely sampled $\left(N_{\text {obs }} \sim\right.$ 1000) $B_{\mathrm{M}}$ and $R_{\mathrm{M}}$ light curves were obtained for a random sample of MACHO LMC RRLs. For each point in the light curves we calculate $\Delta B_{\mathrm{M}}=B_{\mathrm{M}, i}-\left\langle B_{\mathrm{M}}\right\rangle$ and $\Delta R_{\mathrm{M}}=R_{\mathrm{M}, i}-\left\langle R_{\mathrm{M}}\right\rangle$. These are plotted in Figure 7. The line $\Delta B_{\mathrm{M}}=1.32 \Delta R_{\mathrm{M}}$ shows a classic signature of pulsation, namely, that the amplitude of pulsation increases with decreasing wavelength. To compare these points to the observed variation in our objects we determine the fraction of MACHO points enclosed within an error ellipse centered on $\left(\Delta B_{\mathrm{M}}, \Delta R_{\mathrm{M}}\right)$ in Figure 7 for each epoch. $\mathcal{L}_{\text {not var }}$ measures the probability that a nonvariable star of given magnitudes $B_{\mathrm{M}}, R_{\mathrm{M}}$ and photometric uncertainties $\sigma_{B}, \sigma_{R}$ could have the observed variation. $\mathcal{L}_{\text {not var }}$ for the $i$ th observation is expressed as follows:

$$
\mathcal{L}_{\text {not var, } i}=\frac{1}{2 \pi \sigma_{B} \sigma_{R}} e^{-1 / 2\left[R_{\mathrm{M}, i}-\left\langle R_{\mathrm{M}}\right\rangle / \sigma_{R}\right]^{2}} e^{-1 / 2\left[B_{\mathrm{M}, i}-\left\langle B_{\mathrm{M}}\right\rangle / \sigma_{B}\right]^{2}} .
$$

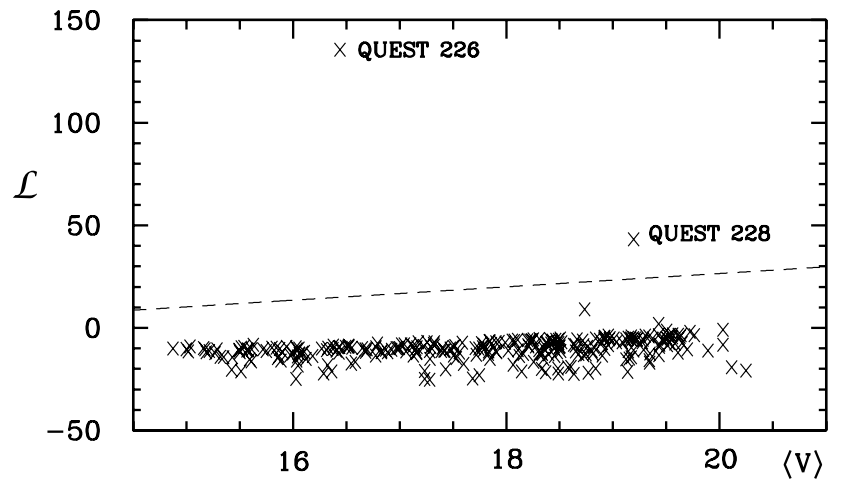

FIG. 8.- RRL index $\mathcal{L}$ vs. $V$ magnitude for one of the survey fields that overlaps the QUEST (Vivas \& Zinn 2006) survey. The two QUEST RRLs in the field are recovered above the dashed line that separates the non-RRLs of low $\mathcal{L}$ from the candidates with high $\mathcal{L}$.

The combined RRL index, $\mathcal{L}$, for a typical field is shown in Figure 8 . Of the 3928 objects identified in this field only 523 objects pass the shape and color restrictions and remain in this figure. The bulk of stars without RRL-like variability and color have indices less than zero. Two objects in the field in question show high indices. We identify RRL candidates above the dashed line in Figure 8. This line is derived from the distribution of 20 QUEST RRLs in our survey area (all are RRab stars). Of these RRLs, eight reside in the nonvariable locus and 12 present $\mathcal{L}>40$. The line slopes upward with $V$ to avoid the flaring of the stellar locus at fainter magnitudes. Candidate RRLs were then visually inspected to remove objects associated with obvious image flaws (ghosting, bad columns, proximity to bright stars, etc.). We also limit candidate colors to $V-R<0.3$, as discussed in $\S 7$. This resulted in 2016 RRL candidates, listed in Table 2.

\section{SURVEY COMPLETENESS}

The survey completeness is the fraction of RRLs recovered by our detection technique from those available in the field. A detailed characterization of the completeness is necessary for the analysis to follow. The completeness depends critically on magnitude since our ability to recover RRLs decreases as we delve fainter due to increasing photometric uncertainty. In addition, the limiting magnitude varies across our surveyed fields. Therefore it was necessary to calculate the completeness and a function of magnitude for each field.

To measure the completeness we created artificial RRL light curves drawn from the period, amplitude and color distributions of the MACHO LMC RRL sample discussed in $\S 5$. Since RRLab and RRLc stars have different period and amplitude distributions we considered the two populations separately. Layden (1998) template light curves were used to construct the RRLab light curves and sine curves were used for the RRL $c$ stars. The initial phase of the simulated light curve was chosen at random. Subsequent samples of the light curve were made at the intervals of the observations for the field in question to account for the phase coverage of the field in question.

To these idealized light curves we added Gaussian-distributed photometric uncertainties as described by equation (8). To measure the completeness as a function of magnitude we generated artificial light curves in $0.5 \mathrm{mag}$ bins from $14<R_{\mathrm{M}}<21$. The artificial data were passed through the selection pipeline and the recovered fraction formed the completeness estimate for that field and magnitude bin. The artificial data, however, do not suffer from the imposition of bad columns, occasional dead amplifiers 
TABLE 2

Table of RR Lyrae Candidates Found in the Present Work

\begin{tabular}{|c|c|c|c|c|c|c|}
\hline Object ID & $\begin{array}{c}\text { R.A. } \\
\text { (J2000.0) }\end{array}$ & $\begin{array}{c}\text { Decl. } \\
(\mathrm{J} 2000.0)\end{array}$ & $V$ & $V-R$ & $E(V-R)$ & $\begin{array}{l}\text { Heliocentric Distance } \\
(\mathrm{kpc})\end{array}$ \\
\hline $128416.544 \ldots \ldots \ldots \ldots \ldots \ldots$ & 001252.83 & 050256.71 & 17.17 & 0.19 & 0.014 & 20.3 \\
\hline $116375.115 \ldots \ldots \ldots \ldots \ldots \ldots$ & 001328.83 & 020343.68 & 14.50 & 0.08 & 0.015 & 5.9 \\
\hline $128412.1045 \ldots \ldots \ldots \ldots \ldots \ldots$ & 001437.09 & 035127.99 & 18.35 & 0.20 & 0.013 & 35.0 \\
\hline 115608.149 & 001614.73 & 015351.58 & 15.34 & 0.18 & 0.019 & 8.6 \\
\hline
\end{tabular}

Noте.-Table 2 is published in its entirety in the electronic edition of the Astrophysical Journal. A portion is shown here for guidance regarding its form and content. Units of right ascension are hours, minutes, and seconds, and units of declination are degrees, arcminutes, and arcseconds.

and inter-CCD gaps. To correctly account for these features we determine the effective area of each field. The effective area is the area of the field that has science data from each exposure. On average, this is $89 \%$ of the maximum $0.51 \mathrm{deg}^{2}$.

Typical completeness profiles are shown in Figure 9. For RRLab stars our survey averages $\sim 60 \%$ completeness for $V<18.5$. This falls to a mean completeness of $25 \%$ by $V=19.5$, although a handful of fields extend to $V=19.5$ due to a combination of low sky brightness and exceptionally good seeing. The completeness for RRL $c$ stars is systematically lower than that of the RRLab stars due to their lower amplitudes. Not surprisingly, the completeness increases with more observations of the field. Figure 10 shows a map of the completeness over the survey fields. Fields with anomalous low completeness typically have bad photometry or are derived from observations that are separated by a few minutes and are thus insensitive to the timescale of RRL variation.

The overlap between our survey and QUEST provides a check on our determined completeness. As stated above, of the 20 QUEST RRLab stars in our survey area, 12 are recovered. This is a magnitude-averaged completeness of $60 \%$. This compares reassuringly well with the average completeness expected from these fields of 55\%. In the case of RRL $c$ stars the numbers

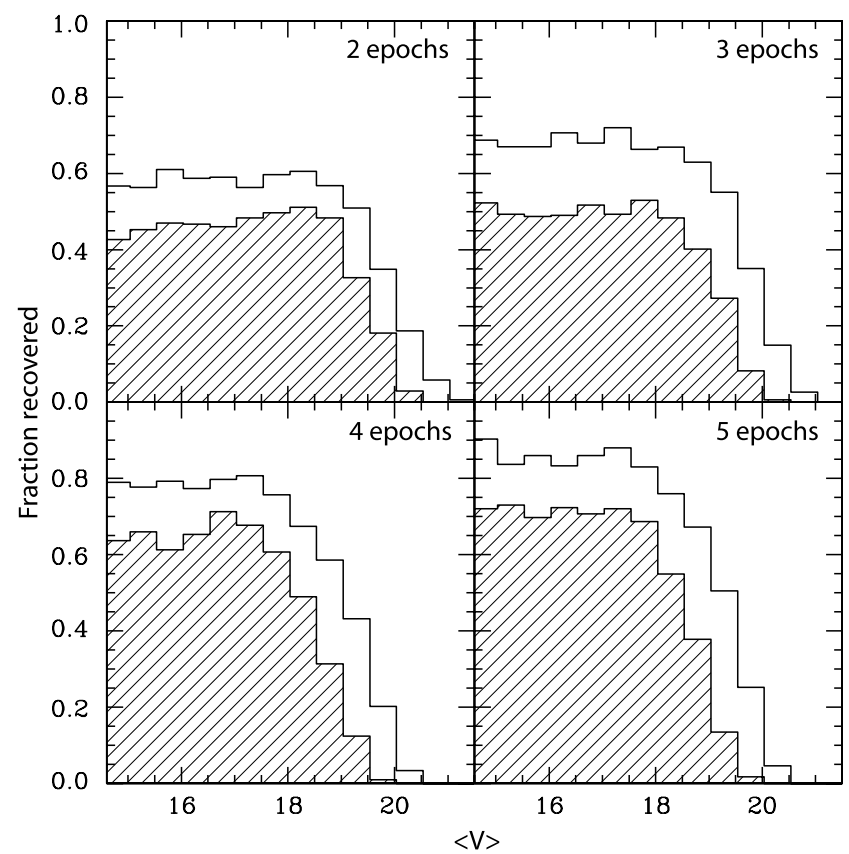

FIG. 9.- Typical completeness profiles as a function of $V$ magnitude for fields with $2,3,4$, and 5 epochs ( $a-d$, respectively). The shaded histograms represent RRL type $c$ stars. are too small to draw any conclusions; one was recovered out of three expected.

\subsection{Magnitude Offsets}

The limited number of observations and the asymmetric shape of the RRLab light curve introduces a bias in the mean magnitude of selected candidates. This can be understood quite simply. Due to the shape of the RRLab light curve less time is spent near maximum light. Yet an RRLab has highest likelihood of selection (highest $\mathcal{L}$ ) when observed at maximum and then again at minimum. This introduces a net bias toward brighter mean magnitudes among the RRLs selected as candidates than would be derived from well sampled light curves of the same stars. For bright RRLs the offset is minimal-these objects are detected with high significance from variations that were only a small fraction of the full amplitude due to the low photometric uncertainties. As we proceed to fainter candidates, the fraction of the full amplitude that must be exhibited for the object to stand out as a variable increases as the photometric uncertainty rises.

Since the photometric uncertainty as a function of magnitude varies from field to field we construct the mean magnitude offset for each individual candidate. A series of artificial RRLab light curves was generated at the magnitude of the candidate as discussed above. We then determined the offset between the mean magnitude and the mean recovered from the observational epochs. The mean magnitude offset for the ensemble of RRL candidates is shown in Figure 11. RRLc stars do not exhibit any significant bias due to their symmetric light curves. Since we do not know

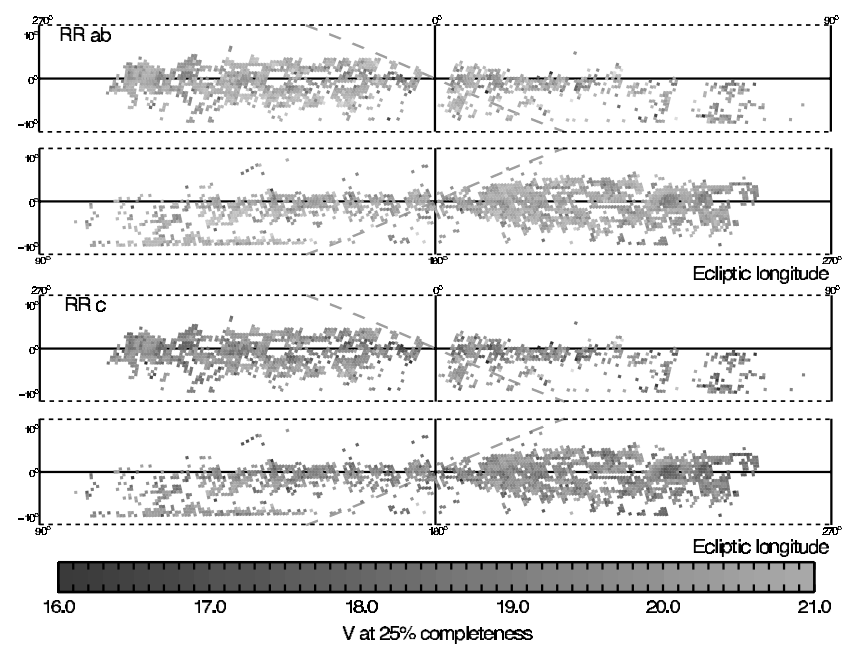

FIG. 10.-Map of completeness across the survey. The color represents the $V$ magnitude at which the completeness drops to $25 \%$ in the case of the RRab (top two panels) and RRc (bottom two panels) variables. The celestial equator is shown as the inclined dashed line. 


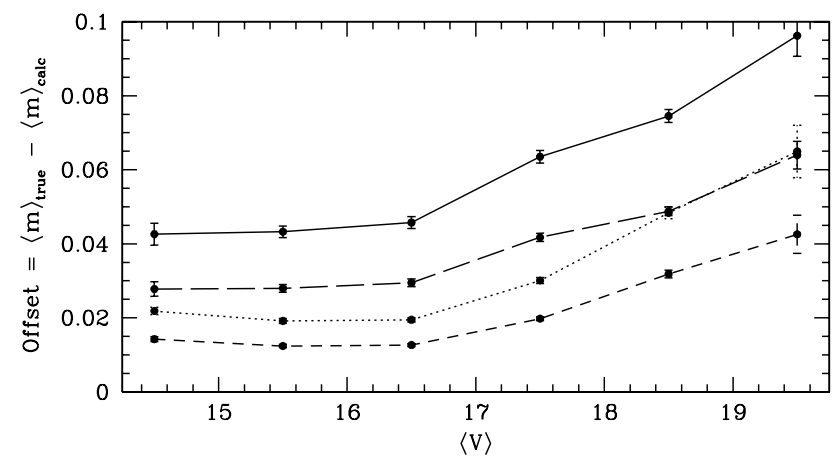

FIG. 11.-Asymmetric nature of the RRLab light curve introduces a magnitude dependent offset to the mean magnitude derived for our RRL candidates. The mean magnitude offsets for $B_{\mathrm{M}}$ (fields with two observations, solid line; fields with more than two observations, dotted line) and $R_{\mathrm{M}}$ (the long-dashed line for two observations; short-dashed line for more than two) are shown as a function of magnitude for the ensemble of RRL candidates.

a priori whether a candidate is $\mathrm{ab}$ or $\mathrm{c}$ type we weight the mean magnitude offset by the fraction of each Bailey type to the RRL population. We use a ratio of $\mathrm{RRab}$ to $\mathrm{RR} c$ of $91: 9$ ratio (Smith 1995), although this fraction varies with the Oosterhoff type of the system. For example, in the case of the Draco dSph (OoI) $\mathrm{RR} c$ contribute $11 \%$ of the 146 known RRLs (Bonanos et al. 2004) whereas in Boötes dSph (OoII) they comprise 7 of 15 known RRLs (Siegel 2006). An OoII halo with $50 \%$ RR $c$ would mean that we have systematically underestimated the brightness of each object by at most 0.05 mag toward our faint limit.

\section{SURVEY CONTAMINATION}

In order to address the level of contamination by non-RRL objects we performed follow-up observations of a sample of $66 \mathrm{RRL}$ candidates with the Wide-Field Imager on the RSAA 40 inch $(1.01 \mathrm{~m})$ telescope at Siding Spring Observatory. The selected candidates spanned a range of magnitudes and $V-R$ color. Light curves of between 8 and 16 points per star were obtained and examined for periodicity (see S. Prior et al. 2008, in preparation). The break down of this sample as a function of color is shown in Figure 12. Those that could be phased were either RRLs or eclipsing binaries. A small number of objects were clearly variable yet unclassifiable, and a similar number showed no significant variation over the 4-6 nights of observation.

In the analysis to follow, we apply a color cut to the RRL candidates of $V-R<0.3$. This cut represents a $2 \sigma$ limit of the MACHO LMC RRL color distribution. With such a color cut we find 16 non-RRLs among 66 candidates (i.e., a contamination rate of $24 \% \pm 12 \%$ ).

\section{PROPERTIES OF THE RR LYRAE CANDIDATES}

The selection process discussed above results in $2016 \mathrm{RRL}$ candidates. Figure 13 shows the color-magnitude diagram for the candidate RRLs relative to the general halo background. The halo color-magnitude diagram shows a steep drop-off in density for stars of $V-R<0.3$; this is the color of the halo main sequence turnoff. The distribution of candidates is bound to the red by our color limit at $V-R=0.3$ and to the blue is consistent with the distribution of MACHO LMC RRLs.

We adopt an absolute magnitude of $M_{V}=0.56$ for the RRL population. This value is based on the mean absolute magnitude of $M_{V}=0.59 \pm 0.03$ for an $[\mathrm{Fe} / \mathrm{H}]=-1.5$ provided by Cacciari \& Clementini (2003), who reviewed the absolute magnitude determinations for RRL from a variety of techniques. The mean metallicity of a sample of halo RRLs from Suntzeff et al. (1991)

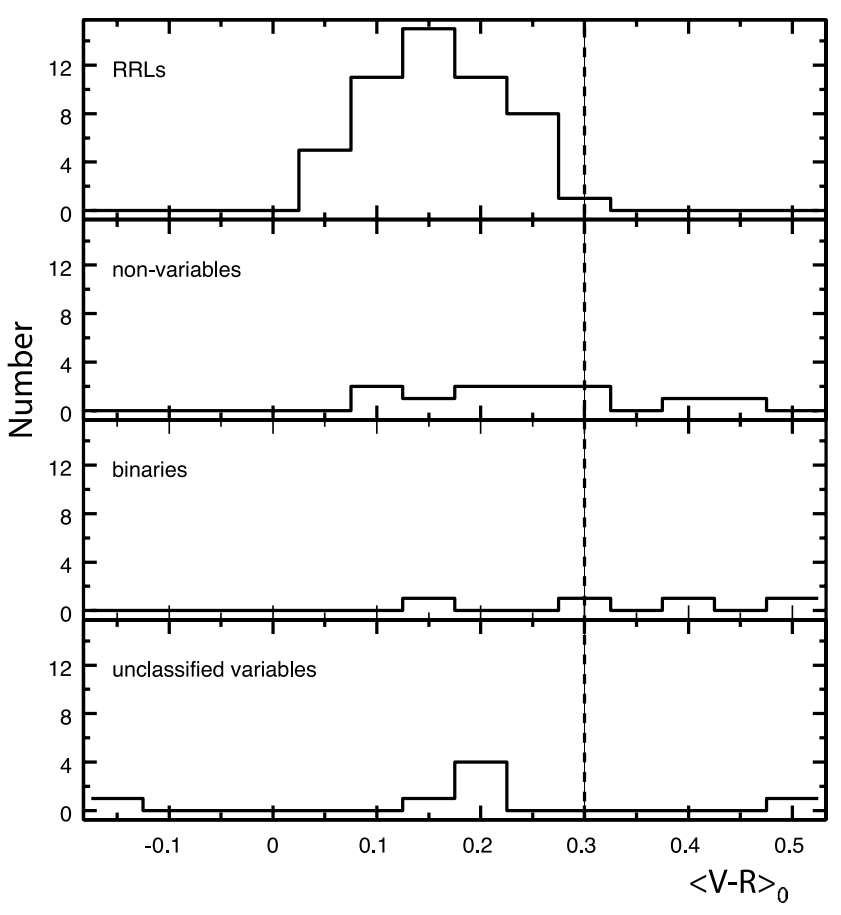

FIG. 12.-Classification of 66 RRL candidates obtained from follow-up photometry.

is $[\mathrm{Fe} / \mathrm{H}]=-1.65 \pm 0.3 \mathrm{dex}$; therefore, we apply an offset to the value of Cacciari \& Clementini (2003) using the metallicity relation of Chaboyer (1999). Our assumed absolute magnitude is equivalent to that used in the study of Vivas \& Zinn (2006), who find that the effects of evolution away from the zero-age horizontal branch and dispersion in metallicity should lead to a dispersion in $M_{V}$ of $0.13 \mathrm{mag}$, or in other words a $7 \%$ dispersion in distance.

\subsection{Spatial and Radial Distribution of RR Lyrae Stars}

In Figure 14 we present the radial distribution of RRL candidates. We collapse the distribution in ecliptic latitude, the natural system for our sample. A series of overdensities can be seen, in particular, at ecliptic latitude $120^{\circ}, 190^{\circ}$, and $320^{\circ}$. These features are, however, overshadowed by the spatial nonuniformity of the survey and the general density falloff away from the Galactic center. In the next two sections we characterize the general candidate background in order to subtract it and reveal the significance of these features.

Figure 15 shows the position of these overdensities on the plane of the sky in the context of known significant substructure. We now discuss these features in more detail:

\subsection{Spatial Density of RRL Candidates}

It is clear from the substructure evident in Figure 14 that the halo does not exhibit a smooth radial density profile. In order to characterize the significance of the observed substructure we determined the general halo background on which the substructures are overlaid.

Note that our sample includes significant contamination by non-RRL objects. It is unlikely that the contaminants share the spatial distribution or absolute magnitude of the RRL population. In the analysis that follows we treat each object as an RRL. The effect this has on the derived spatial density of candidates depends on the nature of the contaminants. For example, mainsequence eclipsing binaries of significantly lower absolute magnitudes than RRLs will appear over a range of magnitudes out to 


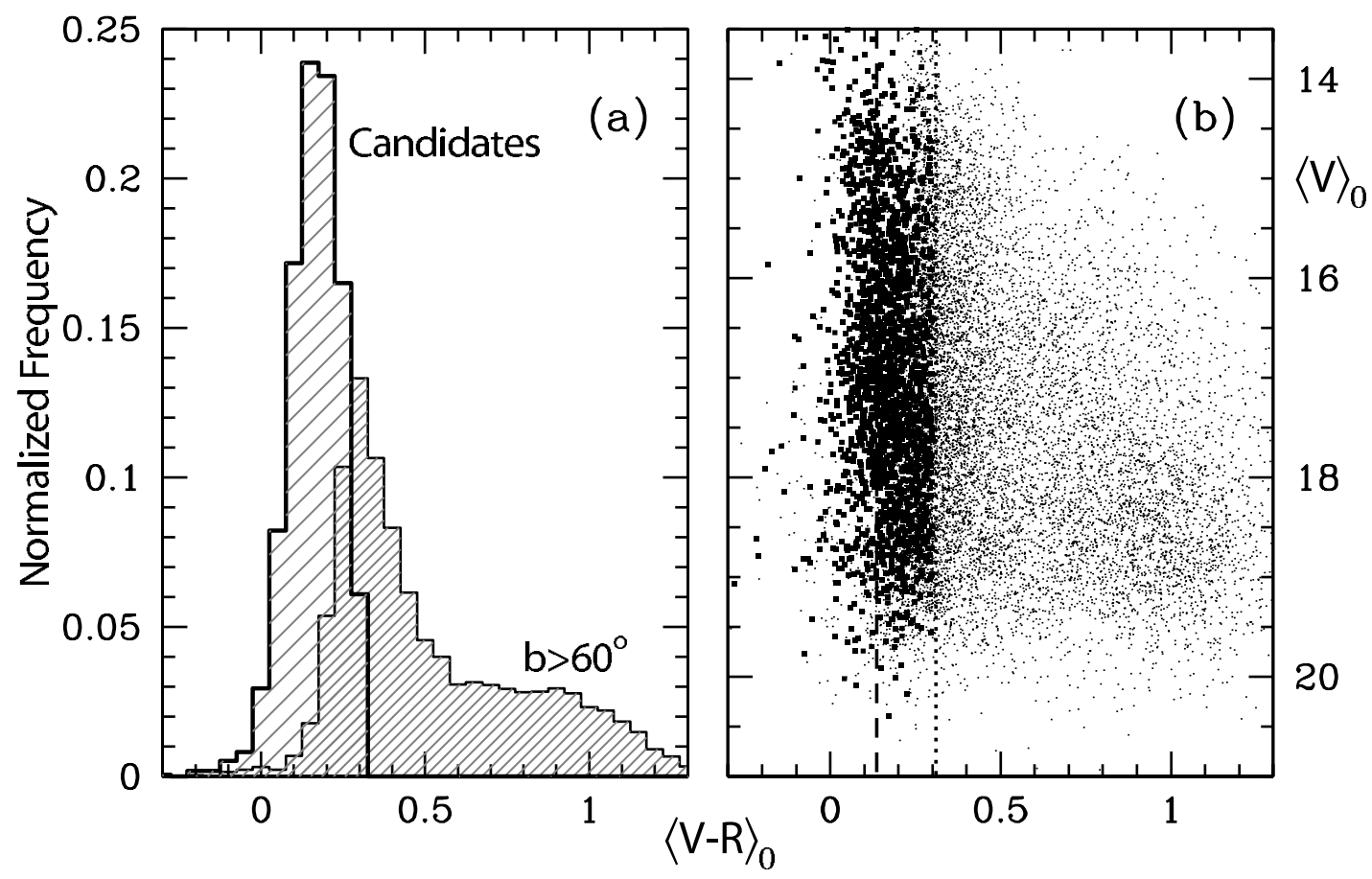

FIG. 13.- (a) Normalized distribution of candidate RRL colors compared to that of a representative sample of $\sim 11,000$ dereddened $b>60^{\circ}$ halo stars. $(b)$ The corresponding color-magnitude diagram for the candidate sample and halo population. The long dashed line is the mean $V-R$ of the MACHO RRL distribution discussed in $\S 5$. The dotted line at $V-R=0.3$ is the imposed color cut.

the edge of the disk along a line of sight. Assuming RRL absolute magnitudes for these objects will act to enhance the space density at large distances. The goal of this exercise is not, however, to derive the spatial density of RRLs but rather to derive a model for the background halo.

There are two key models discussed in the literature for the radial distribution of matter in the halo. General ellipsoidal isopycnic contours for the halo can be expressed as

$$
\frac{x^{2}}{a^{2}}+\frac{y^{2}}{b^{2}}+\frac{z^{2}}{c^{2}}=1 \text {. }
$$

The spherical model $(a=b=c)$ is the most basic, in which the density varies as a function of galactocentric distance as a power law,

$$
\rho(R)=\rho_{0}\left(R / R_{0}\right)^{n}
$$

where $\rho_{0}$ is the local space density of RRLs and $R_{0}$ is the solar radius.

The case of a spheroidal halo has $a=b$ and flattening described by $q \equiv c / a$. Numerous observational studies have found that the inner $<20 \mathrm{kpc}$ of the halo is significantly flattened while
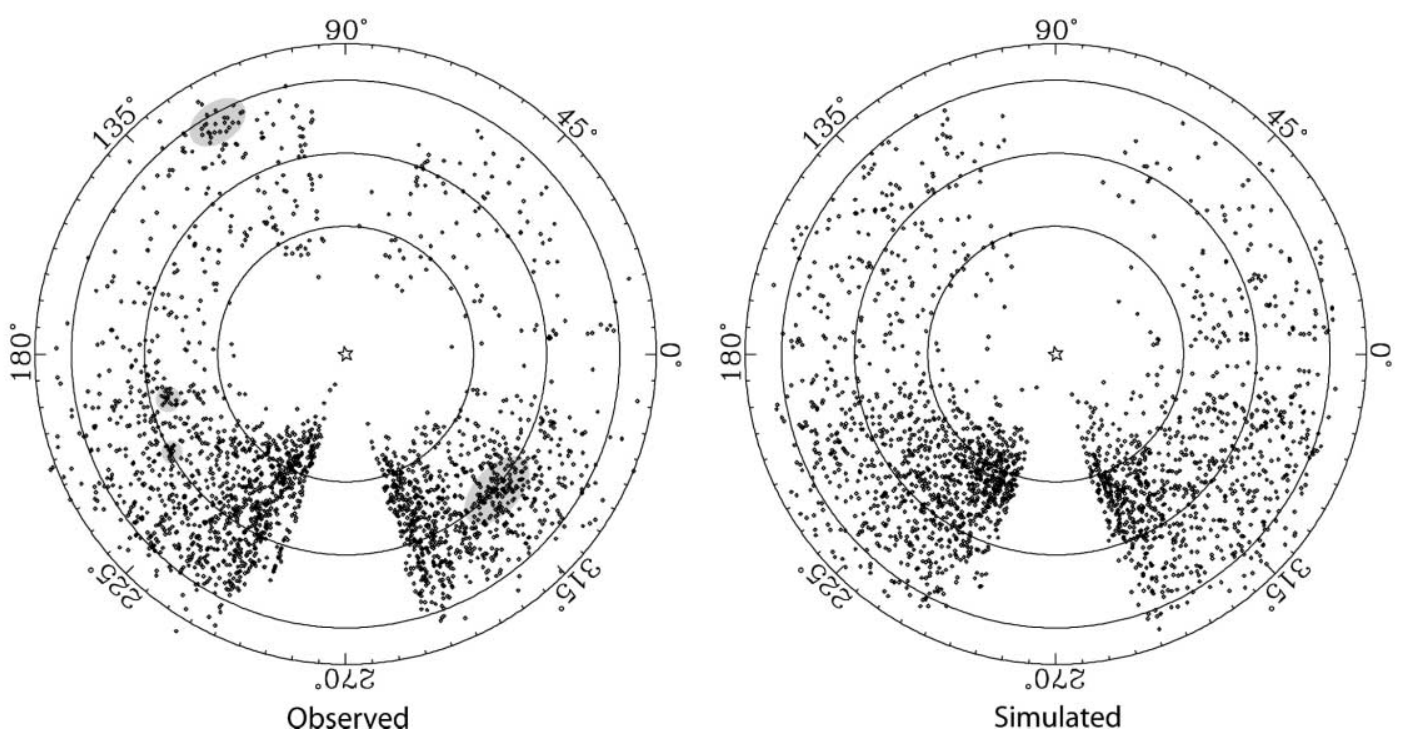

FIG. 14. - Left: The radial distribution of RRL candidates in ecliptic longitude. Right: The corresponding distribution of objects from one of our Monte Carlo simulations of the halo (see $\S 8.3$ ). A number of substructures (shaded in figure) are apparent in the observations that are not found in the simulation, in particular at ecliptic latitude $120^{\circ}, 190^{\circ}, 210^{\circ}$, and $320^{\circ}$. The significance of these structures is discussed in $\S 8.3$. The circles are placed at $V=15,17$, and 19 . 


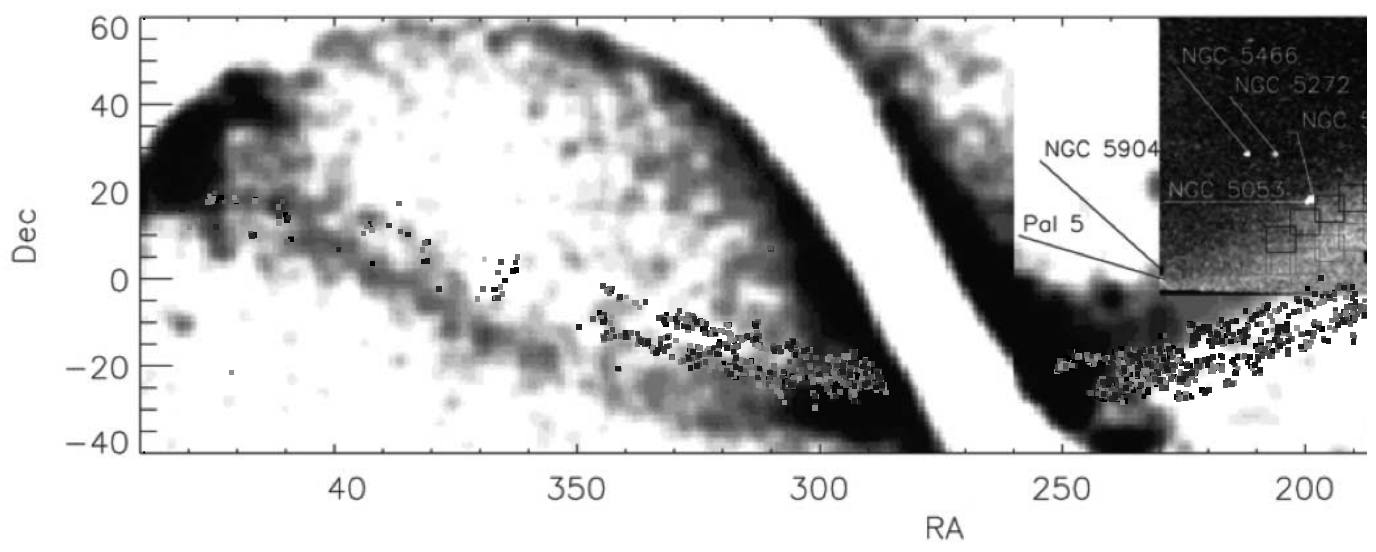

FIG. 15.- Our 2016 RRL candidates color coded by distance overlaid on the view of the outer halo due to Belokurov et al. (2007), which includes analysis of the main-sequence turnoff stars from SDSS (top right insert) and M giants from the study of Majewski et al. (2003) (the grey-scale background). The Sagittarius stream dominates the view of the outer halo (the main body of the Sagittarius dwarf is located centrally in this figure at decl. $\sim-20^{\circ}$. [See the electronic edition of the Journal for a color version of this figure.]

the outer regions appear spherical (Chiba \& Beers 2000; Preston et al. 1991). The spatially variable flattening ratio introduced by Preston et al. (1991) expresses isopycnic contours as ellipsoids of revolution. Such surfaces vary as a function of the semimajor axis $a$,

$$
c / a= \begin{cases}(c / a)_{0}+\left[1-(c / a)_{0}\right]\left(a / a_{u}\right), & a<a_{u}, \\ 1, & a>a_{u},\end{cases}
$$

where $a_{u}=20 \mathrm{kpc}$ and canonically, $(c / a)_{0}=0.5$. In this case, density varies as a function of semimajor axis as

$$
\rho(a)=\rho_{0}\left(a / R_{0}\right)^{n}
$$

Numerous studies of the RRL distribution have found that the observed distribution is best fit by variable flattening model. However, the difference in the quality of the resultant fit between the spherical and variably flattened models is of little (Vivas \& Zinn 2006) or no (Miceli et al. 2008) statistical significance. In the following we only consider the case of a spherical halo.

The calculation of the RRL space density follows the technique developed by Wetterer \& McGraw (1996). The following equation describes the density as a function of galactocentric distance:

$$
\rho(R)=\frac{1}{4 \pi R^{2} f(R)} \frac{d N}{d R},
$$

where $f(R)$ is the fraction of the total halo volume at $R$ that is sampled by the survey $(f(R)$ is analogous to a solid angle) and $\mathrm{N}$ is the number of RRL as a function of distance. Whereas the solid angle is constant as one looks through the halo, $f(R)$ varies as a function of Galactocentric radius and hence must be calculated numerically for each field. This is because the volume of each field is a pyramid subtending the field's effective area (see $\S 6$ ) at infinity and an apex offset from the Galactic center by $R_{0}$. Different lines of sight sample different regions of the Galactocentric sphere. This is demonstrated for two lines of sight in Figure 16. One field is of higher Galactic latitude and samples more of the inner Galactic halo. To generate these profiles we calculate $R$ and the volume of each slice of the pyramid for a given $r+d r$. The volumes are summed into $0.1 \mathrm{kpc}$ bins before being divided by the volume of the sphere at the radius $R$ of the bin. As $R$ tends to large values $f(R)$ approaches the asymptotic limit of (effective area) $/ 4 \pi$, corresponding to the constant solid angle as would occur if $R_{0}=0$.

To account for the effects of completeness we multiply each $f(R)$ by the Monte-Carlo derived RRLab completeness profile for the field as a function of galactocentric radius. This gives the effective volume of the halo that is sampled by each field (Fig. 16, right-hand plot). The total volume of our sample is then simply the sum of $f(R)$ for the 3692 individual fields. This is shown in Figure 17.

We then use equation (16) to calculate the local space density for each candidate. To distill the space density of our sample we then bin space densities of the 2016 RRL candidates in bins of 0.02 dex in $\log (R)$. The resulting average density distribution is shown in Figure 18. The error bars in Figure 18 represent the standard deviation of the points in each bin. Since 50-30 objects reside in a typical bin the large standard deviation is indicative of intrinsic azimuthal variability in the space density such as would result from substructure.

As stated above, our goal in the examination of the average space density is to extract the mean background on which the substructure lies. A linear least squares fit to points between $10<R<45 \mathrm{kpc}$ yields $n=-2.48 \pm 0.09$ and $\rho_{0}=(6.22 \pm$ 0.12 ) $\mathrm{kpc}^{-3}$ (as introduced in eq. [12]). Beyond $R=45 \mathrm{kpc}$ there

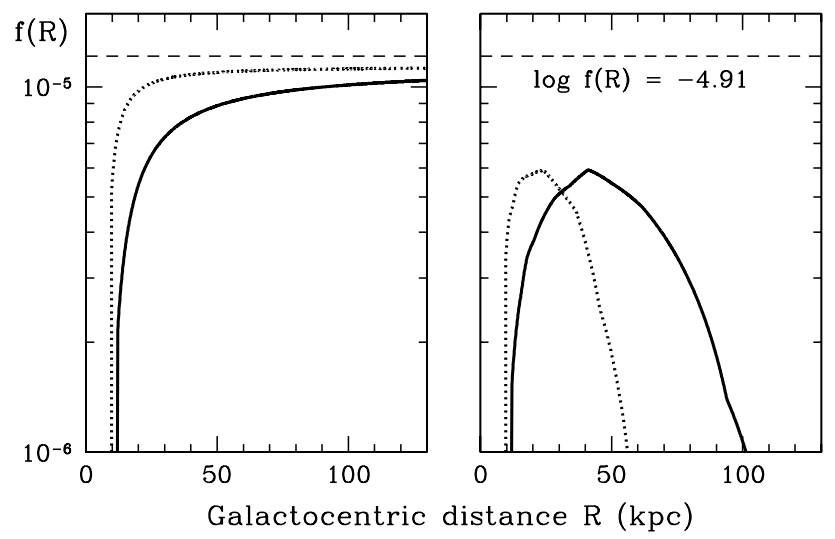

FIG. 16. - Variation of the fractional halo volume as a function of Galactocentric distance, $f(R)$. Left panel: For two fields centered on $(l, b)=\left(213^{\circ}, 34^{\circ}\right)$ (solid line) and $\left(262^{\circ}, 60^{\circ}\right)$ (dotted line). The asymptotic limit represents the constant solid angle subtended by the fields if $R_{0}=0$ with infinite limiting magnitude. Right panel: The same profiles corrected for completeness as a function of $R$. The profiles for all fields are summed to give the total $f(R)$ for the survey. 


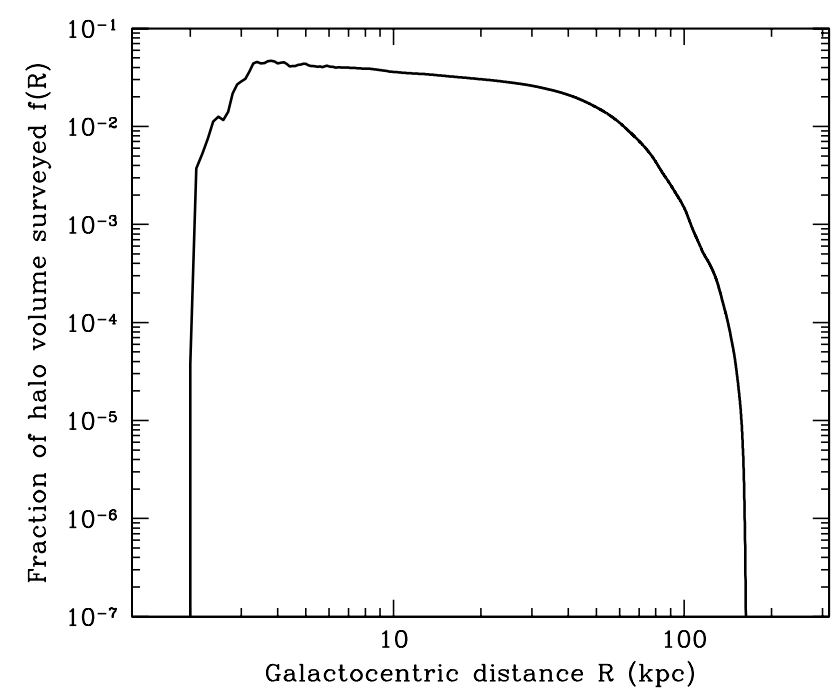

FIG. 17.- Total fractional volume of the halo surveyed by the 3692 fields.

is a more rapid drop in the number of candidate RRLs. For the 122 candidates with $R>45 \mathrm{kpc}$ we find a $n=-5.0 \pm 0.3$ power law. We plan to follow-up $R>45 \mathrm{kpc}$ candidates to see if the RRLs among them match this increased radial drop-off. However, as stated before, since the candidates contain significant contamination by non-RRLs, we do not interpret these results further at present but rather use them as the basis of our detection of spatial departures from the mean background.

\subsection{Halo Substructure}

In this section we quantify the significance of substructure in the halo. We firstly construct a series of artificial halos based on the spatial distribution function discussed above. This is complicated in the case of our survey by nonuniform spatial, temporal, and radial coverage; however, all three are well characterized for each field. To form a simulated halo we first randomly select a position in the sky from the area covered by our survey fields. We then use the spatial density power-law indices discussed above to randomly assign a distance and then use the completeness information for the field in which it lies to see whether we would recover such a candidate. This is repeated until the total number of candidates observed is recovered. An example of a simulated halo is shown in Figure 14.

We next perform a Voronoi tessellation (Voronoi 1907; Ramella et al. 2001) of the simulated halo distribution in (heliocentric distance, ecliptic longitude) space. The Voronoi tessellation associates with each point a polygon such that every point inside the polygon is closest to the point in question. It enables us to define the local density of points in a nonparametric way as simply the reciprocal of the area of the tesserae. We repeat this for the entire set of simulated halos and form a map of the mean density of simulated candidates in (distance, ecliptic longitude) space. Along with the mean we determine a map of the standard deviation in the simulated density. From the Voronoi tessellation of the observed RRL candidate distribution we subtract the mean density of simulated candidates and divide by the standard deviation to obtain a map of the significance of spatial structure in the halo as seen in Figure 19.

\subsubsection{Sagittarius Dwarf Debris Streams}

In the interpretation of Figure 19 it is useful to consider the position of the orbital plane of the Sagittarius dwarf galaxy that features prominently in the outer halo (as seen in Fig. 15). Our

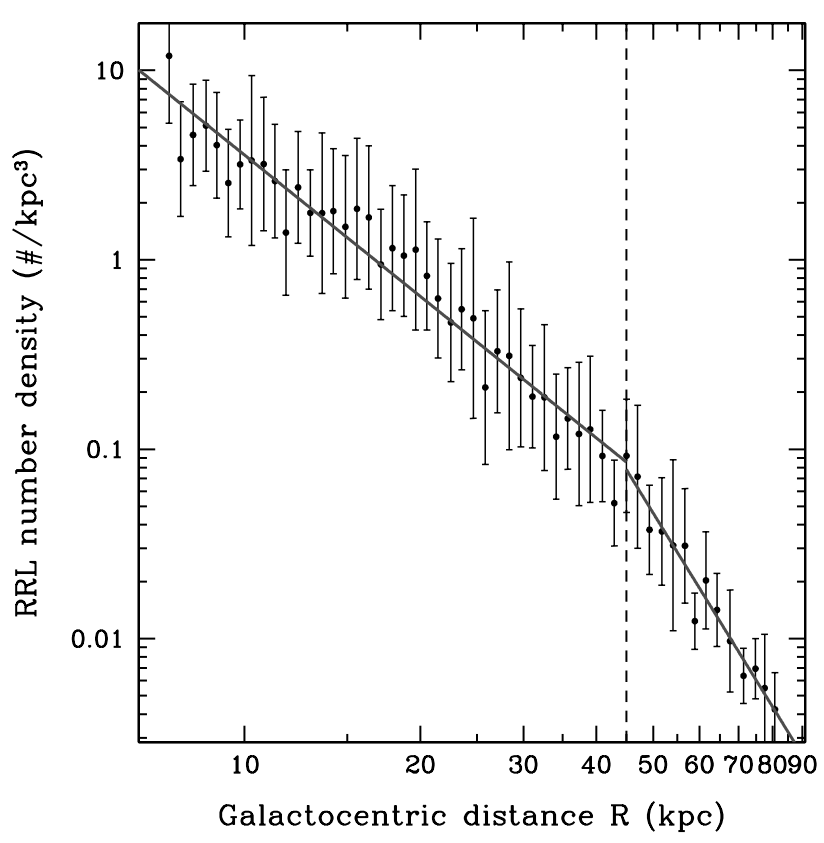

FIG. 18.-Apparent spatial density of RRL candidates determined assuming that all candidates are RRLs (see text for discussion of the best-fit lines). We use this result to quantify the average background on which substructure resides.

survey cuts obliquely through the plane of the Sagittarius dwarf (Sgr; as defined by Ibata et al. 2001) at an angle of $16^{\circ}$ with an opening angle of $10^{\circ}$ (see Fig. 19). We obtain maximum signal from structures in the Sagittarius stream at angles adjacent to the line of nodes. The structures from R.A. $=45^{\circ}-130^{\circ}$ and $220^{\circ}-330^{\circ}$ lie in this range. In Figure 19 we show a view of the Sagittarius tidal arms compiled from the observations of Majewski et al. (2003) M giants, Belokurov et al. (2006) F dwarfs and Newberg et al. (2003); Newberg et al. (2007) Blue horizontal branch stars and $\mathrm{F}$ dwarfs together with the model predictions of Helmi \& White (2001), Law et al. (2005), and Fellhauer et al. (2006).

The models of Law et al. (2005) and Helmi (2004) aim to match the observations of M giants (Majewski et al. 2003). Helmi (2004) finds that the data strongly suggests that the dark matter halo of the Milky Way is prolate $[1.25<(q=a / c)<1.5]$. Law et al. (2005) similarly require the presence of a significantly prolate halo $(q=1.25)$ but could not establish a model that reproduces both the observed orbital pole precession and the distance and radial velocity of the leading arm. Johnston et al. (2005) conclude that it is necessary to consider an evolution in the orbital parameters of Sgr over the time frame of the last few orbits ( $\sim 2-3$ Gyr) and propose that dynamical friction is the most favorable mechanism to bring about this change.

Fellhauer et al. (2006) models the Sgr system in the light of the Belokurov et al. (2006) result. In particular, Fellhauer et al. seeks to interpret the observed bifurcation of the Sgr stream. This work is based on the distances derived to the F dwarf population. As pointed out by Newberg et al. (2003) a 13\% discrepancy in distance exists between the distances derived from $M$ giants and those from $\mathrm{F}$ dwarfs. The $\mathrm{M}$ giant distance scale has proven to be systematically smaller than the F dwarf scale and it is unclear which is more accurate. Fellhauer et al. finds that the observed bifurcation is only possible in a near-spherical halo and that it is not possible to match the distances and radial velocities of the leading arm in their closest approach to the solar circle. We must bear in mind these caveats to the modeling of the debris from Sgr 

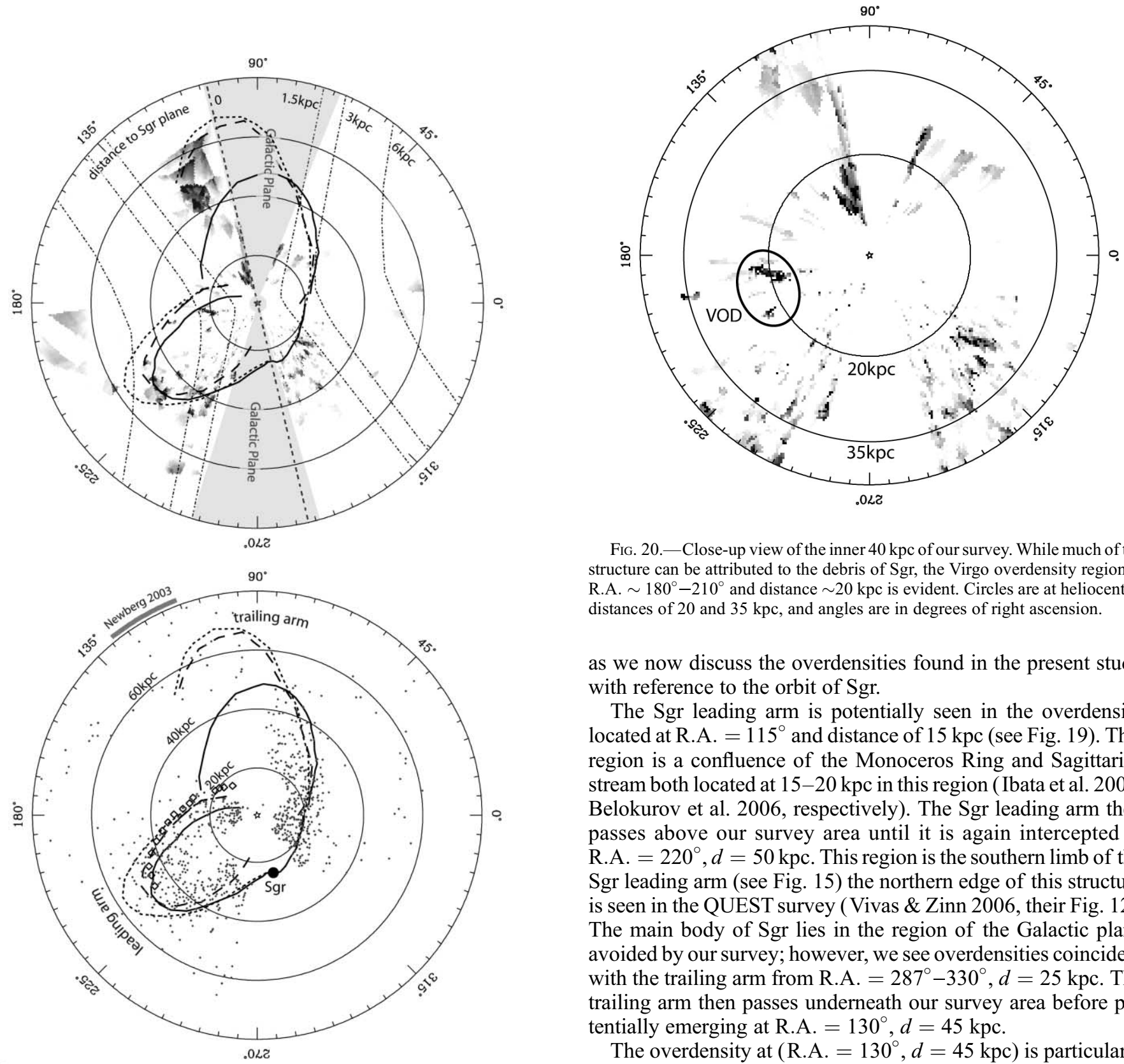

FIG. 20.-Close-up view of the inner $40 \mathrm{kpc}$ of our survey. While much of the structure can be attributed to the debris of Sgr, the Virgo overdensity region at R.A. $\sim 180^{\circ}-210^{\circ}$ and distance $\sim 20 \mathrm{kpc}$ is evident. Circles are at heliocentric distances of 20 and $35 \mathrm{kpc}$, and angles are in degrees of right ascension.

as we now discuss the overdensities found in the present study with reference to the orbit of Sgr.

The Sgr leading arm is potentially seen in the overdensity located at R.A. $=115^{\circ}$ and distance of $15 \mathrm{kpc}$ (see Fig. 19). This region is a confluence of the Monoceros Ring and Sagittarius stream both located at 15-20 kpc in this region (Ibata et al. 2003; Belokurov et al. 2006, respectively). The Sgr leading arm then passes above our survey area until it is again intercepted at R.A. $=220^{\circ}, d=50 \mathrm{kpc}$. This region is the southern limb of the Sgr leading arm (see Fig. 15) the northern edge of this structure is seen in the QUEST survey (Vivas \& Zinn 2006, their Fig. 12). The main body of Sgr lies in the region of the Galactic plane avoided by our survey; however, we see overdensities coincident with the trailing arm from R.A. $=287^{\circ}-330^{\circ}, d=25 \mathrm{kpc}$. The trailing arm then passes underneath our survey area before potentially emerging at R.A. $=130^{\circ}, d=45 \mathrm{kpc}$.

The overdensity at (R.A. $=130^{\circ}, d=45 \mathrm{kpc}$ ) is particularly interesting. It is separated by $\sim 200^{\circ}$ from the main body of Sgr along the trailing arm. Other than the overdensity of A-type stars detected by Newberg et al. (2003) this overdensity is the most distant and oldest portion of Sgr debris yet found and offers a critical constraint on the orbit of Sgr and hence the potential of the Galaxy in which it moves. As we can see from the divergence between the models of Law et al. (2005) and Fellhauer et al. (2006) in this region (see Fig. 19, middle panel), older material offers the strongest constraint on the model orbit. However, radial velocities for stars in the observed overdensities are required to confirm their association with Sgr. We are now obtaining radial velocities for RR Lyrae stars found along the debris stream. By confronting the radial velocity predictions of dynamical models with observations we hope to converge on increasingly accurate models for the Galactic halo and orbital elements for Sgr.

\subsubsection{Virgo Overdensity}

The region between R.A. $=180^{\circ}$ and $230^{\circ}$ (seen in Fig. 20) exhibits two significant overdensities, one at R.A. $=12^{\mathrm{h}} 47^{\mathrm{m}}$, 


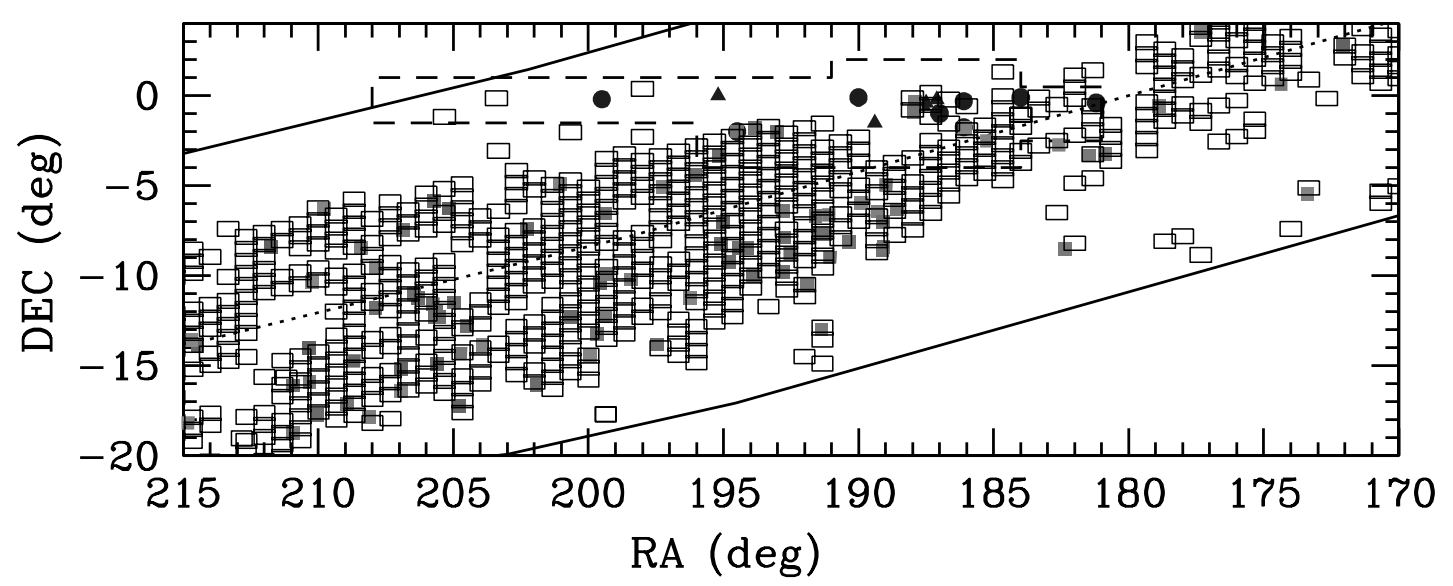

FIG. 21.-Distribution of RRL candidates in the region of the Virgo overdensity covered by our survey (survey fields are shown by open squares). The points shown have heliocentric distances of $16<r<26 \mathrm{kpc}$. Clump 1 is seen at R.A. $=192^{\circ}$, decl. $=-8^{\circ}$, and clump 2 at R.A. $=206^{\circ}$, decl. $=-12^{\circ}$. The sample of RRLs and blue horizontal branch stars of Duffau et al. (2006) are shown as filled circles and triangles, respectively. The dashed outline shows the region where Duffau et al. found an excess of main-sequence stars of appropriate apparent magnitude to be associated with the Virgo overdensity.

decl. $=-06^{\circ} 42^{\prime}$ and distance of $16 \mathrm{kpc}$ and a second at R.A. $=$ $13^{\mathrm{h}} 47^{\mathrm{m}}$, decl. $=-11^{\circ} 24^{\mathrm{m}}$ and a distance of $19 \mathrm{kpc}$. Both lie in the direction of several previously identified halo substructures. The first indication of substructure in this region was presented by Newberg et al. (2002) region S297+63-20.5; at a distance of $18 \mathrm{kpc}$. Zinn et al. (2004) found a significant overdensity from their RRL survey (distance of $19 \mathrm{kpc}$ ). Subsequent spectroscopy of a sample of RRLs and blue horizontal branch stars from within this clump revealed a moving group with $V_{\mathrm{gsr}}=100 \pm 13 \mathrm{~km} \mathrm{~s}^{-1}$ (Duffau et al. 2006 dubbed the Virgo stellar stream). Jurić et al. (2005) used photometric parallaxes of SDSS stars and found a broad overdensity centered on the above (the Virgo overdensity [VOD]) that covers over a thousand square degrees between distances of 5-15 kpc. Martínez-Delgado et al. (2007) suggests that the leading arm of tidal debris from the Sagittarius dwarf, as modeled by Law et al. (2005) could provide the excess required to account for the VOD. Newberg \& Yanny (2005) have suggested the VOD may result from a nonaxisymmetric component to the Galaxy such as a triaxial halo.

Newberg et al. (2007) determines radial velocities for a sample of F stars drawn from the S297+63-20.5 region and finds radial velocities of $V_{\mathrm{gsr}}=130 \pm 10 \mathrm{~km} \mathrm{~s}^{-1}$. The low velocity dispersion of these stars is inconsistent with their origin in a triaxial halo and their radial velocity is at odds with that expected from stars in the Sgr leading tidal tail. Newberg et al. (2007) proposes that the three named features are one, we use the term Virgo overdensity hereafter to refer to this single system.

The first overdensity, VOD clump 1 , lies $8^{\circ}$ southwest of the region identified by Duffau et al. (2006) as shown in Figure 21. This is followed by a second overdensity $16^{\circ}$ further to the southwest, VOD clump 2. It is apparent from the results of Belokurov et al. (2006) and Newberg et al. (2007) that regardless of the likely shape of the VOD the central regions must lie out of the SDSS region in the southern hemisphere. The twin clumps found by the present study plausibly form structure in the core of the VOD. However, we must point out that due to the nonuniform survey coverage we are unable to clearly define spatial substructure. We are in the process of attaining radial velocities for samples from the two clumps to verify if they do indeed share the systemic velocity found by Duffau et al. (2006) for the VOD.

\section{SUMMARY}

In this paper we have presented a survey for RR Lyrae stars that has revealed a sample of 2016 RR Lyrae candidates from multiple (2-10) epoch data covering $1675 \mathrm{deg}^{2}$. Follow up photometric observations of these candidates show $76 \%$ to be RR Lyrae variables. Our survey reveals a series of significant overdensities in the Galactic halo. Many of the overdense regions lie along the leading and trailing arms of debris from the Sagittarius dwarf galaxy extending up to $\sim 200^{\circ}$ from the main body of the Sagittarius dwarf. Another locality of overdensity cannot be ascribed to the Sagittarius dwarf: namely, the region of the Virgo overdensity. Here we find two significant overdensities $10^{\circ}$ to the southwest of the center previously reported.

Radial velocities of RR Lyrae stars drawn from our overdensities will give insight into the dynamics and origins of these systems. In particular, regions coincident with the leading and trailing arms of the Sagittarius dwarf could offer powerful constraints on the orbit and evolution of the Sagittarius dwarf.

We thank Ken Freeman for his comments that helped to improve this paper. This data is based on observations from Mount Stromlo Observatory's $1.3 \mathrm{~m}$ telescope before its untimely demise in the bushfire of 2003 January 18. This paper utilizes public domain data obtained by the MACHO Project, jointly funded by the US Department of Energy through the University of California, Lawrence Livermore National Laboratory, under contract W-7405-Eng-48, by the National Science Foundation through the Center for Particle Astrophysics of the University of California under cooperative agreement AST 88-09616, and by the Mount Stromlo and Siding Spring Observatory, part of the Australian National University. This publication makes use of data products from the Two Micron All Sky Survey, which is a joint project of the University of Massachusetts and the Infrared Processing and Analysis Center/California Institute of Technology, funded by the National Aeronautics and Space Administration and the National Science Foundation.

Facilities: MtS:1.3m. 
Abadi, M. G., Navarro, J. F., \& Steinmetz, M. 2006, MNRAS, 365, 747

Alcock, C., et al.1999, PASP, 111, 1539

2004, AJ, 127, 334

Bell, E. F., et al. 2007, ApJ, submitted (arXiv: 0706.0004)

Belokurov, V., et al. 2006, ApJ, 642, L137 2007, ApJ, 657, L89

Bertin, E. 2005, SWarp User's Guide (ver. 2.15.4), Institut d'Astrophysique \& Observatoire de Paris

Bessell, M. S., \& Germany, L. M. 1999, PASP, 111, 1421

Bonanos, A. Z., Stanek, K. Z., Szentgyorgyi, A. H., Sasselov, D. D., \& Bakos, G. Á. 2004, AJ, 127, 861

Bullock, J., \& Johnson, K. 2005, ApJ, 635, 931

Bullock, J. S., Kravtsov, A. V., \& Weinberg, D. H. 2001, ApJ, 548, 33

Cacciari, C., \& Clementini, G. 2003, in Stellar Candles for the Extragalactic Distance Scale, ed. D. Alloin \& W. Gieren (Berlin: Springer), 105

Calabretta, M. R., Valdes, F., Greisen, E. W., \& Allen, S. L. 2004, in ASP Conf. Ser. 314, Astronomical Data Analysis Software and Systems) XIII, ed. F. Ochsenbein, M. G. Allen, \& D. Egret (San Francisco: ASP), 551

Carollo, D., et al. 2007, Nature, 450, 1020

Casetti-Dinescu, D. I., Majewski, S. R., Girard, T. M., Carlin, J. L., van Altena, W. F., Patterson, R. J., \& Law, D. R. 2006, AJ, 132, 2082

Chaboyer, B. 1999, in Post-Hipparcos Cosmic Candles, ed. A. Heck \& F. Caputo (Dordrecht: Kluwer), 111

Chiba, M., \& Beers, T. C. 2000, AJ, 119, 2843

Conn, B. C., et al. 2007, MNRAS, 376, 939

Duffau, S., Zinn, R., Vivas, A. K., Carraro, G., Méndez, R. A., Winnick, R., \& Gallart, C. 2006, ApJ, 636, L97

Eggen, O. J., Lynden-Bell, D., \& Sandage, A. R. 1962, ApJ, 136, 748

Fellhauer, M., et al. 2006, ApJ, 651, 167

Freeman, K., \& Bland-Hawthorn, J. 2002, ARA\&A, 40, 487

Helmi, A. 2004, ApJ, 610, L97

Helmi, A., \& White, S. D. M. 2001, MNRAS, 323, 529

Ibata, R. A., Gilmore, G., \& Irwin, M. J. 1994, Nature, 370, 194

Ibata, R. A., Irwin, M. J., Lewis, G. F., Ferguson, A. M. N., \& Tanvir, N. 2003, MNRAS, 340, L21

Ibata, R., Lewis, G. F., Irwin, M., Totten, E., \& Quinn, T. 2001, ApJ, 551, 294

Ivezić, Ž., et al. 2000, AJ, 120, 963 2004, in ASP Conf. Ser. 327, Satellites and Tidal Streams, ed. F. Prada, D. Martinez Delgado, \& T. J. Mahoney (San Francisco: ASP), 104 Ivezić, Ž., Vivas, A. K., Lupton, R. H., \& Zinn, R. 2005, AJ, 129, 1096 Johnston, K. V., Law, D. R., \& Majewski, S. R. 2005, ApJ, 619, 800 Jurić, M., et al. 2005, preprint (astro-ph/0510520)

Kinman, T. D., Cacciari, C., Bragaglia, A., Buzzoni, A., \& Spagna, A. 2007, MNRAS, 375, 1381
Landolt, A. U. 1992, AJ, 104, 340

Law, D. R., Johnston, K. V., \& Majewski, S. R. 2005, ApJ, 619, 807

Layden, A. C. 1998, AJ, 115, 193

Lee, J.-W., \& Carney, B. W. 1999, AJ, 118, 1373

Majewski, S. R., Skrutskie, M. F., Weinberg, M. D., \& Ostheimer, J. C. 2003, ApJ, 599, 1082

Martin, N. F., Rodrigo, A., Ibata, R. A., \& Irwin, M. 2007, ApJ, 668, L123

Martínez-Delgado, D., Peñarrubia, J., Jurić, M., Alfaro, E. J., \& Ivezić, Z. 2007, ApJ, 660, 1264

Miceli, A., et al. 2008, ApJ, 678, 865

Moody, R., Schmidt, B., Alcock, C., Goldader, J., Axelrod, T., Cook, K. H., \& Marshall, S. 2003, Earth Moon Planets, 92, 125

Newberg, H. J., \& Yanny, B. 2005, in ASP Conf. Serl. 338, Astrometry in the Age of the Next Generation of Large Telescopes, ed. P. K. Seidelmann \& A. K. B. Monet (San Francisco: ASP), 210

Newberg, H. J., Yanny, B., Cole, N., Beers, T. C., Re Fiorentin, P., Schneider, D. P., \& Wilhelm, R. 2007, ApJ,. 668, 221

Newberg, H. J., et al. 2003, ApJ, 596, L191

2002, ApJ, 569, 245

Preston, G. W., Shectman, S. A., \& Beers, T. C. 1991, ApJ, 375, 121

Ramella, M., Boschin, W., Fadda, D., \& Nonino, M. 2001, A\&A, 368, 776

Rocha-Pinto, H. J., Majewski, S. R., Skrutskie, M. F., Crane, J. D., \& Patterson, R. J. 2004, ApJ, 615, 732

Schlegel, D. J., Finkbeiner, D. P., \& Davis, M. 1998, ApJ, 500, 525

Searle, L., \& Zinn, R. 1978, ApJ, 225, 357

Sesar, B. 2007, AJ, 134, 2236

Siegel, M. H. 2006, ApJ, 649, L83

Skrutskie, M. F., et al. 2006, AJ, 131, 1163

Smith, H. A. 1995, RR Lyrae Stars (Cambridge: Cambridge Univ. Press)

Stetson, P. B. 2000, PASP, 112, 925

Suntzeff, N. B., Kinman, T. D., \& Kraft, R. P. 1991, ApJ, 367, 528

Vivas, A. K., \& Zinn, R. 2006, AJ, 132, 714

Voronoi, G. 1907, J. Reine Angewandte Math., 133, 97

Wetterer, C. J., \& McGraw, J. T. 1996, AJ, 112, 1046

Wilkinson, M. I. 2007, in The Milky Way Halo-Stars and Gas, ed. K. de Boer \& P. Kroupa (Univ. Bonn), http://www.astro.uni-bonn.de/ mwhalo/proceedings/ mwh-t53-wilkinson.pdf

Yanny, B.,et al. 2003, ApJ, 588, 824

Zacharias, N., Urban, S. E., Zacharias, M. I., Wycoff, G. L., Hall, D. M., Monet, D. G., \& Rafferty, T. J. 2004, AJ, 127, 3043

Zinn, R., Vivas, A. K., Gallart, C., \& Winnick, R. 2004, in ASP Conf. Ser. 327, Satellites and Tidal Streams, ed. F. Prada, D. Martinez Delgado, \& T. J. Mahoney (San Francisco: ASP), 92 